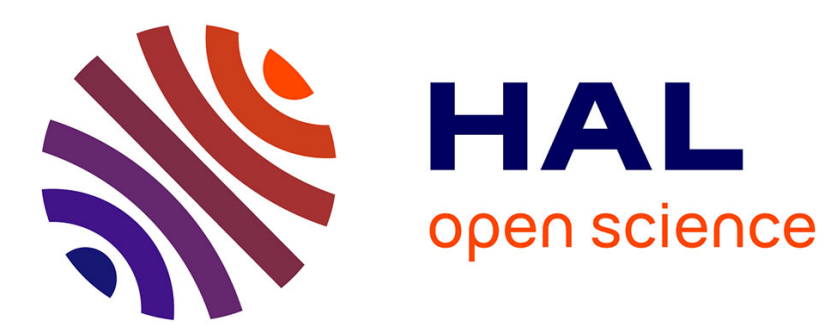

\title{
An unconditionally stable uncoupled scheme for a triphasic Cahn-Hilliard/Navier-Stokes model
}

\author{
Sebastian Minjeaud
}

\section{To cite this version:}

Sebastian Minjeaud. An unconditionally stable uncoupled scheme for a triphasic CahnHilliard/Navier-Stokes model. Numerical Methods for Partial Differential Equations, 2013, 29 (2), pp. 584- 618 . 10.1002/num.21721. hal-00577226v2

\section{HAL Id: hal-00577226 \\ https://hal.science/hal-00577226v2}

Submitted on 21 Oct 2011

HAL is a multi-disciplinary open access archive for the deposit and dissemination of scientific research documents, whether they are published or not. The documents may come from teaching and research institutions in France or abroad, or from public or private research centers.
L'archive ouverte pluridisciplinaire $\mathbf{H A L}$, est destinée au dépôt et à la diffusion de documents scientifiques de niveau recherche, publiés ou non, émanant des établissements d'enseignement et de recherche français ou étrangers, des laboratoires publics ou privés. 


\title{
AN UNCONDITIONALLY STABLE UNCOUPLED SCHEME FOR A TRIPHASIC CAHN-HILLIARD/NAVIER-STOKES MODEL
}

\author{
SEBASTIAN MINJEAUD ${ }^{\dagger \ddagger}$
}

\begin{abstract}
We propose an original scheme for the time discretization of a triphasic CahnHilliard/Navier-Stokes model. This scheme allows an uncoupled resolution of the discrete CahnHilliard and Navier-Stokes system, is unconditionally stable and preserves, at the discrete level, the main properties of the continuous model. The existence of discrete solutions is proved and a convergence study is performed in the case where the densities of the three phases are the same.
\end{abstract}

Key words. Cahn-Hilliard/Navier-Stokes model; Time discretization; Unconditional stability.

AMS subject classifications. 35K55, 65M60, 65M12, 76T30.

1. Introduction. The complexity of multiphasic flows basically lies in the fact that the time evolution of interfaces, whose position is an unknown of the problem, may lead to their deformation, their break-up or coalescence. Moreover, interfaces obey to physical phenomena where capillary effects play an important role.

The various domains of application, where multiphasic flows are involved, are generally complex; the experimentation and measurements are quite difficult, onerous and most often not very accurate. For instance, in nuclear safety [23], the understanding of interaction between molten corium (lava-like molten mixture of portions of nuclear reactor core) and concrete (last confinement barrier) is a major issue. An approach using direct numerical simulations allows to access to instantaneous quantities at each point of the flows.

Because of their ability to capture interfaces implicitly, diffuse interfaces models are attractive for the numerical simulations of multiphase flows. In this article, we consider a model which couples the Cahn-Hilliard system and the Navier-Stokes equation.

1.1. The Cahn-Hilliard model. In diffuse interfaces theory, the interfaces are assumed to have a non-zero thickness $\varepsilon$ (which is here a constant parameter of the model). Interfaces are considered as mixing areas and the phase $i$ can be represented by a smooth phase indicator $c_{i}$ called order parameter (which may be understood here as the volumic fraction of the phase $i$ ). Thus, the system contains as many unknowns $c_{i}$ as phases. These unknowns vary between 0 and 1 (values which correspond to pure phases by convention) and are linked by the relationship $\sum_{i} c_{i}=1$.

A complete derivation of this kind of model for diphasic flows is presented in references [1], [2], [17] or [20]. Different extensions have been proposed for the simulations of three-phase flows in references [4], [11] or [19]. We consider in this article the triphasic Cahn-Hilliard model taken from reference [4]:

$$
\left\{\begin{aligned}
\frac{\partial c_{i}}{\partial t} & =\operatorname{div}\left(\frac{M_{0}(\mathbf{c})}{\Sigma_{i}} \nabla \mu_{i}\right), & & \text { for } i=1,2,3 \\
\mu_{i} & =f_{i}^{F}(\mathbf{c})-\frac{3}{4} \varepsilon \Sigma_{i} \Delta c_{i}, & & \text { for } i=1,2,3
\end{aligned}\right.
$$

\footnotetext{
${ }^{\dagger}$ Institut de Radioprotection et de Sûreté Nucléaire, Cadarache, France

‡Laboratoire d’Analyse, Topologie et Probabilités, Université Paul Cézanne, Marseille, France
} 
where $M_{0}(\mathbf{c})$ is a diffusion coefficient which is called mobility and may depend on $\mathbf{c}=\left(c_{1}, c_{2}, c_{3}\right)$. The functions $f_{i}^{F}$ are defined by:

$$
f_{i}^{F}(\mathbf{c})=\frac{4 \Sigma_{T}}{\varepsilon} \sum_{j \neq i}\left(\frac{1}{\Sigma_{j}}\left(\partial_{i} F(\mathbf{c})-\partial_{j} F(\mathbf{c})\right)\right)
$$

where $\Sigma_{T}$ is given by $\frac{3}{\Sigma_{T}}=\frac{1}{\Sigma_{1}}+\frac{1}{\Sigma_{2}}+\frac{1}{\Sigma_{3}}$. This system is a gradient flow for the following energy functional under the constraint of volume conservation:

$$
\mathcal{F}_{\boldsymbol{\Sigma}, \varepsilon}^{\mathrm{triph}}\left(c_{1}, c_{2}, c_{3}\right)=\int_{\Omega} \frac{12}{\varepsilon} F\left(c_{1}, c_{2}, c_{3}\right)+\frac{3}{8} \varepsilon \sum_{i=1}^{3} \Sigma_{i}\left|\nabla c_{i}\right|^{2} d x,
$$

where $\Omega$ denote an open, bounded, connected and smooth domain of $\mathbb{R}^{d}(d=2$ or $d=3$ ). The "intermediate" unknowns $\mu_{i}$, called chemical potentials, are the functional derivatives of the triphasic Cahn-Hilliard energy (1.2). The rather intricate expression of $f_{i}^{F}$ let us ensure the constraint:

$$
c_{1}+c_{2}+c_{3}=1
$$

We introduce the hyperplane $\mathcal{S}=\left\{\left(c_{1}, c_{2}, c_{3}\right) \in \mathbb{R}^{3} ; c_{1}+c_{2}+c_{3}=1\right\}$ of $\mathbb{R}^{3}$, to simplify notation in the sequel.

The expressions of the triphasic Cahn-Hilliard potential $F$ and of the constant triplet $\boldsymbol{\Sigma}=\left(\Sigma_{1}, \Sigma_{2}, \Sigma_{3}\right)$ was derived in [4], so that the model can correctly take into account the surface tensions values $\sigma_{12}, \sigma_{13}$ and $\sigma_{23}$ prescribed between the different pairs of phases and so that it is consistent with the two-phase situations: the triphasic model has to exactly reproduce diphasic situations when one of the three phases is not present. The coefficient $\Sigma_{i}$ is given as a function of the surface tensions:

$$
\Sigma_{i}=\sigma_{i j}+\sigma_{i k}-\sigma_{j k}, \quad \forall i \in\{1,2,3\} .
$$

and the triphasic potential $F$ has the following form:

$$
\begin{aligned}
F(\mathbf{c})=\sigma_{12} c_{1}^{2} c_{2}^{2}+\sigma_{13} c_{1}^{2} c_{3}^{2} & +\sigma_{23} c_{2}^{2} c_{3}^{2} \\
& +c_{1} c_{2} c_{3}\left(\Sigma_{1} c_{1}+\Sigma_{2} c_{2}+\Sigma_{3} c_{3}\right)+c_{1}^{2} c_{2}^{2} c_{3}^{2} \Lambda(\mathbf{c}), \quad \forall \mathbf{c} \in \mathcal{S},
\end{aligned}
$$

where $\Lambda$ is an arbitrary smooth function of $\mathbf{c}$.

Note that, in the sequel, we do not assume that the coefficients $\Sigma_{i}$ are non negative, so that the model can handle some total spreading situations. However, as it is proved in [4], the following condition is necessary to ensure the well-posedness of the system:

$$
\Sigma_{1} \Sigma_{2}+\Sigma_{1} \Sigma_{3}+\Sigma_{2} \Sigma_{3}>0
$$

This condition is equivalent to the coercivity of capillary terms and ensures that these terms bring a positive contribution to the free energy $\mathcal{F}_{\Sigma, \varepsilon}^{\text {triph }}$. This is detailed in the following proposition:

Proposition 1.1 ([4, Prop 2.1]). Let $\boldsymbol{\Sigma}=\left(\Sigma_{1}, \Sigma_{2}, \Sigma_{3}\right) \in \mathbb{R}^{3}$. There exists $\underline{\Sigma}>0$ such that, for all $n \geq 1$, for all $\left(\boldsymbol{\xi}_{\mathbf{1}}, \boldsymbol{\xi}_{\mathbf{2}}, \boldsymbol{\xi}_{\mathbf{3}}\right) \in\left(\mathbb{R}^{n}\right)^{3}$ such that $\boldsymbol{\xi}_{\mathbf{1}}+\boldsymbol{\xi}_{\mathbf{2}}+\boldsymbol{\xi}_{\mathbf{3}}=0$,

$$
\Sigma_{1}\left|\boldsymbol{\xi}_{\mathbf{1}}\right|^{2}+\Sigma_{2}\left|\boldsymbol{\xi}_{\mathbf{2}}\right|^{2}+\Sigma_{3}\left|\boldsymbol{\xi}_{3}\right|^{2} \geqslant \underline{\Sigma}\left(\left|\boldsymbol{\xi}_{1}\right|^{2}+\left|\boldsymbol{\xi}_{\mathbf{2}}\right|^{2}+\left|\boldsymbol{\xi}_{\mathbf{3}}\right|^{2}\right)
$$


if and only if the two following conditions are satisfied:

$$
\Sigma_{1} \Sigma_{2}+\Sigma_{1} \Sigma_{3}+\Sigma_{2} \Sigma_{3}>0 \text { and } \Sigma_{i}+\Sigma_{j}>0, \forall i \neq j .
$$

This proposition will be useful in the sequel.

ReMARK 1. Owing to (1.3), the second part of condition (1.5) is always satisfied and consequently it is sufficient to assume that the condition (1.4) holds, for applying proposition 1.1.

The existence of weak solutions for problem (1.1) together with initial and Neumann boundary conditions (for order parameters $c_{i}$ and chemical potentials $\mu_{i}$ ) was proved in [4] (see [7] for an alternative proof based a numerical schemes) in 2D and $3 \mathrm{D}$ under the following general assumptions:

- the mobility $M_{0}$ is a bounded function of $\mathcal{C}^{1}\left(\mathbb{R}^{3}\right)$ class and there exists three positive constants $M_{1}, M_{2}$ and $M_{3}$ such that:

$$
\forall \mathbf{c} \in \mathcal{S}, \quad 0<M_{1} \leqslant M_{0}(\mathbf{c}) \leqslant M_{2}, \quad \text { and } \quad\left|D M_{0}(\mathbf{c})\right| \leqslant M_{3} .
$$

- the Cahn-Hilliard potential $F$ is a positive function of $\mathcal{C}^{2}\left(\mathbb{R}^{3}\right)$ class which satisfies the following assumptions of polynomial growth: there exist a constant $B_{1}>0$ and a real $p$ such that $2 \leqslant p<+\infty$ for $d=2$ or $2 \leqslant p \leqslant 6$ for $d=3$, and

$$
\begin{gathered}
\forall \mathbf{c} \in \mathcal{S}, \quad|F(\mathbf{c})| \leqslant B_{1}\left(1+|\mathbf{c}|^{p}\right), \quad|D F(\mathbf{c})| \leqslant B_{1}\left(1+|\mathbf{c}|^{p-1}\right), \\
\text { and }\left|D^{2} F(\mathbf{c})\right| \leqslant B_{1}\left(1+|\mathbf{c}|^{p-2}\right) .
\end{gathered}
$$

1.2. Coupling with hydrodynamic. The coupling between the Cahn-Hilliard and Navier-Stokes systems is obtained by:

1. adding a transport term $\mathbf{u} \cdot \nabla c_{i}$ in the evolution equation of each order parameter $c_{i},(i \in\{1,2,3\})$, that is the first equation of system (1.1).

2 . defining the density and viscosity as smooth functions of order parameters $\mathbf{c}$.

3. adding a capillary forces term $\sum_{i=1}^{3} \mu_{i} \nabla c_{i}$ in the right hand side of the momentum balance (in the Navier-Stokes equation).

Furthermore, we adopt a particular form of the Navier-Stokes equation, initially proposed in [15] (see also [6] and [20]), which ensures an energy balance without using the equation of mass conservation. It relies on the following inequality:

$$
\frac{d}{d t} \int_{\Omega_{t}} \frac{1}{2} \varrho|\mathbf{u}|^{2} d x=\int_{\Omega_{t}}\left[\sqrt{\varrho} \frac{\partial}{\partial t}(\sqrt{\varrho} \mathbf{u})+(\varrho \mathbf{u} \cdot \nabla) \mathbf{u}+\frac{\mathbf{u}}{2} \operatorname{div}(\varrho \mathbf{u})\right] \cdot \mathbf{u} d x
$$

the domain $\Omega_{t}$ being an arbitrary bounded smooth domain moving at the fluid velocity $\mathbf{u}[3]$. 
Hence, the triphasic Cahn-Hilliard/Navier-Stokes, we study here, is constituted with following equations:

$$
\left\{\begin{array}{l}
\frac{\partial c_{i}}{\partial t}+\mathbf{u} \cdot \nabla c_{i}=\operatorname{div}\left(\frac{M_{0}}{\Sigma_{i}} \nabla \mu_{i}\right), \quad \forall i=1,2,3 \\
\mu_{i}=\frac{4 \Sigma_{T}}{\varepsilon} \sum_{j \neq i}\left(\frac{1}{\Sigma_{j}}\left(\partial_{i} F(\mathbf{c})-\partial_{j} F(\mathbf{c})\right)\right)-\frac{3}{4} \varepsilon \Sigma_{i} \Delta c_{i}, \quad \forall i=1,2,3 \\
\sqrt{\varrho(\mathbf{c})} \frac{\partial}{\partial t}(\sqrt{\varrho(\mathbf{c})} \mathbf{u})+(\varrho(\mathbf{c}) \mathbf{u} \cdot \nabla) \mathbf{u}+\frac{\mathbf{u}}{2} \operatorname{div}(\varrho(\mathbf{c}) \mathbf{u}) \\
\quad-\operatorname{div}(2 \eta(\mathbf{c}) D(\mathbf{u}))+\nabla p=\sum_{i=1}^{3} \mu_{i} \nabla c_{i}+\varrho(\mathbf{c}) \mathbf{g} \\
\operatorname{div} \mathbf{u}=0,
\end{array}\right.
$$

where the vector $\mathbf{g}$ stands for the gravity; the density and viscosity are defined by:

$$
\varrho(\mathbf{c})=\frac{\sum_{i=1}^{3} \varrho_{i} h_{\lambda}\left(c_{i}-0.5\right)}{\sum_{i=1}^{3} h_{\lambda}\left(c_{i}-0.5\right)} \quad \text { and } \quad \eta(\mathbf{c})=\frac{\sum_{i=1}^{3} \eta_{i} h_{\lambda}\left(c_{i}-0.5\right)}{\sum_{i=1}^{3} h_{\lambda}\left(c_{i}-0.5\right)}
$$

where $\varrho_{1}$ (resp. $\left.\varrho_{2}, \varrho_{3}\right)$ and $\eta_{1}$ (resp. $\left.\eta_{2}, \eta_{3}\right)$ are the values (assumed to be constants) in phase 1 (resp. 2, resp. 3) and the function $h_{\lambda}(\lambda=0.5)$ is defined by:

$$
h_{\lambda}(x)=\left\{\begin{array}{lll}
0 & \text { if } \quad x<-\lambda \\
\frac{1}{2}\left(\frac{x}{\lambda}+\frac{1}{\pi} \sin \left(\pi \frac{x}{\lambda}\right)\right) & \text { if } \quad-\lambda \leqslant x \leqslant \lambda \\
1 & \text { if } \quad x>\lambda .
\end{array}\right.
$$

We supplement this system with Neumann boundary conditions for order parameters $c_{i}$ and chemical potentials $\mu_{i}$, i.e. for $i=1,2,3$,

$$
\nabla c_{i} \cdot \mathbf{n}=0 \text { and } M_{0} \nabla \mu_{i} \cdot \mathbf{n}=0, \text { on } \Gamma
$$

and with a homogeneous Dirichlet boundary condition for the velocity, i.e.

$$
\mathbf{u}=0 \text { on } \Gamma \text {. }
$$

Owing to these boundary conditions (1.9) and (1.10), we introduce the following function spaces:

$$
\begin{aligned}
& \mathcal{V}^{c}=\mathcal{V}^{\mu}=\mathrm{H}^{1}(\Omega), \quad \mathcal{V}_{\mathcal{S}}^{\mathbf{c}}=\left\{\mathbf{c}=\left(c_{1}, c_{2}, c_{3}\right) \in\left(\mathrm{H}^{1}(\Omega)\right)^{3} ; \mathbf{c}(x) \in \mathcal{S} \text { for a. e. } x \in \Omega\right\}, \\
& \mathcal{V}^{\mathbf{u}}=\left(\mathrm{H}^{1}(\Omega)\right)^{d}, \quad \mathcal{V}_{0}^{\mathbf{u}}=\left(\mathrm{H}_{0}^{1}(\Omega)\right)^{d}, \quad \mathcal{V}^{p}=\left\{p \in \mathrm{L}^{2}(\Omega), \int_{\Omega} p d x=0\right\} .
\end{aligned}
$$

Finally, we assume that the following initial condition holds:

$$
c_{i}(t=0)=c_{i}^{0}, \quad \text { and } \quad \mathbf{u}(t=0)=\mathbf{u}^{0},
$$

where $\mathbf{c}^{0}=\left(c_{1}^{0}, c_{2}^{0}, c_{3}^{0}\right) \in \mathcal{V}_{\mathcal{S}}^{\mathbf{c}}$ and $\mathbf{u}^{0} \in \mathcal{V}_{0}^{\mathbf{u}}$ are given. 
1.3. Outline of the article. In section 2, we describe the time and space discretization of problem (1.8). We then prove, in section 3, the unconditional stability of the scheme and the existence of approximate solutions. Section 4 is devoted to numerical experiments. In the last section 5, we prove the convergence of approximate solutions towards weak solutions of the Cahn-Hilliard/Navier-Stokes system in the case where the three fluids have the same density. In particular, we prove the following existence theorem by passing to the limit in the numerical scheme in section 5:

THEOREM 1.2 (Existence of weak solutions in the homogeneous case). Assume the coefficients $\left(\Sigma_{1}, \Sigma_{2}, \Sigma_{3}\right)$ satisfy the condition (1.4), the mobility satisfy (1.6), and that the Cahn-Hilliard potential F satisfy the condition (1.7). Assume the densities of the three fluids are equal, i.e. $\varrho_{1}=\varrho_{2}=\varrho_{3}=\varrho_{0}, \varrho_{0} \in \mathbb{R}$. Consider the problem (1.8) together with initial condition (1.11) and boundary conditions (1.9)-(1.10). Then, for all $\left.t_{f} \in\right] 0,+\infty\left[\right.$, there exists a weak solution $(\mathbf{c}, \boldsymbol{\mu}, \mathbf{u}, p)$ on $\left[0, t_{f}[\right.$ such that

$$
\begin{gathered}
\mathbf{c} \in \mathrm{L}^{\infty}\left(0, t_{f} ;\left(\mathrm{H}^{1}(\Omega)\right)^{3}\right) \cap \mathcal{C}^{0}\left(\left[0, t_{f}\left[;\left(\mathrm{L}^{q}(\Omega)\right)^{3}\right), \text { for all } q<6,\right.\right. \\
\boldsymbol{\mu} \in \mathrm{L}^{2}\left(0, t_{f} ;\left(\mathrm{H}^{1}(\Omega)\right)^{3}\right), \\
\mathbf{u} \in \mathrm{L}^{\infty}\left(0, t_{f} ;\left(\mathrm{L}^{2}(\Omega)\right)^{3}\right) \cap \mathrm{L}^{2}\left(0, t_{f} ;\left(\mathrm{H}^{1}(\Omega)\right)^{3}\right), \\
\mathbf{c}(t, x) \in \mathcal{S}, \text { for almost every }(t, x) \in\left[0, t_{f}[\times \Omega .\right.
\end{gathered}
$$

\section{Discretization of the Cahn-Hilliard/Navier-Stokes model.}

2.1. Time discretization. Let $N \in \mathbb{N}^{*}$. The time domain $\left[0, t_{f}\right]$ is uniformly discretized with a fixed time step $\Delta t=\frac{t_{f}}{N}$; we define $t_{n}=n \Delta t$, for all $n \in \llbracket 0 ; N \rrbracket$. We assume that the function $\mathbf{c}^{n} \in \mathcal{V}_{\mathcal{S}}^{\mathbf{c}}$ and $\mathbf{u}^{n} \in \mathcal{V}_{0}^{\mathbf{u}}(n \in \llbracket 0 ; N-1 \rrbracket)$ are given and we describe the system we have to solve to compute the unknowns $\mathbf{c}^{n+1} \in \mathcal{V}_{\mathcal{S}}^{\mathbf{c}}$ and $\mathbf{u}^{n+1} \in \mathcal{V}_{0}^{\mathbf{u}}$ at time $t^{n+1}$.

We first describe, in two distinct paragraphs, the schemes we use to discretize the Cahn-Hilliard and Navier-Stokes equations without considering the coupling terms. We then explain, in the next two paragraphs, the reasoning leading to the discretization of the coupling terms before writing the complete scheme in the last paragraph. For more details on the time discretizations of the triphasic Cahn-Hilliard model, the reader may refer to the article [7] (and references therein). Several articles in the literature are devoted to the study of discretizations of the Navier-Stokes equation: we refer in particular to the articles [15] and [21] which deal with variable density models.

2.1.1. Cahn-Hilliard system. We consider a time discretization of the CahnHilliard system of the form: for $i=1,2,3$,

$$
\left\{\begin{array}{l}
\frac{c_{i}^{n+1}-c_{i}^{n}}{\Delta t}+\begin{array}{l}
\text { transport } \\
\text { term }
\end{array}=\operatorname{div}\left(\frac{M_{0}^{n+\alpha}}{\Sigma_{i}} \nabla \mu_{i}^{n+1}\right), \\
\mu_{i}^{n+1}=D_{i}^{F}\left(\mathbf{c}^{n}, \mathbf{c}^{n+1}\right)-\frac{3}{4} \varepsilon \Sigma_{i} \Delta c_{i}^{n+\beta}
\end{array}\right.
$$

where $c_{i}^{n+\beta}=(1-\beta) c_{i}^{n}+\beta c_{i}^{n+1}, \beta \in[0.5,1]$, and $M_{0}^{n+\alpha}=M_{0}\left((1-\alpha) \mathbf{c}^{n}+\alpha \mathbf{c}^{n+1}\right)$, $\alpha \in[0,1]$; the discretization of transport terms is postponed to section 2.1.3.

This kind of discretizations was presented and studied in reference [7]. This is out the scope of the article. We assume that the discretization $D_{i}^{F}\left(\mathbf{c}^{n}, \mathbf{c}^{n+1}\right)$ of the 
term $f_{i}^{F}$ is of the form:

$$
D_{i}^{F}\left(\mathbf{a}^{n}, \mathbf{a}^{n+1}\right)=\frac{4 \Sigma_{T}}{\varepsilon} \sum_{j \neq i}\left(\frac{1}{\Sigma_{j}}\left(d_{i}^{F}\left(\mathbf{a}^{n}, \mathbf{a}^{n+1}\right)-d_{j}^{F}\left(\mathbf{a}^{n}, \mathbf{a}^{n+1}\right)\right)\right), \forall\left(\mathbf{a}^{n}, \mathbf{a}^{n+1}\right) \in \mathcal{S}^{2},
$$

where $d_{i}^{F}$ stands for a semi-implicit discretization of $\partial_{i} F$. We assume that the two following basic properties hold for all $i \in\{1,2,3\}$ :

$$
\begin{gathered}
\forall \mathbf{c} \in \mathcal{S}, \quad D_{i}^{F}(\mathbf{c}, \mathbf{c})=f_{i}^{F}(\mathbf{c}) . \\
\forall\left(\mathbf{a}^{n}, \mathbf{a}^{n+1}\right) \in \mathcal{S}^{2}, \quad\left|d_{i}^{F}\left(\mathbf{a}^{n}, \mathbf{a}^{n+1}\right)\right| \leqslant B_{1}\left(1+\left|\mathbf{a}^{n}\right|^{p-1}+\left|\mathbf{a}^{n+1}\right|^{p-1}\right), \\
\left|D\left(d_{i}^{F}\left(\mathbf{a}^{n}, \cdot\right)\right)\left(\mathbf{a}^{n+1}\right)\right| \leqslant B_{1}\left(1+\left|\mathbf{a}^{n}\right|^{p-2}+\left|\mathbf{a}^{n+1}\right|^{p-2}\right),
\end{gathered}
$$

the notation $D$ means here the derivative of $d_{i}^{F}$ with respect to the second variable. The assumption (2.1) is a consistency assumption and the assumption (2.2) is the counterpart of the polynomial growth assumption (1.7) on $F$. Many possible choices for the discretization of the term $d_{i}^{F}$ were presented in [7]. For instance, we consider in numerical tests of this article (section 4 ) the following expression:

$$
\begin{aligned}
& d_{i}^{F_{0}}\left(\mathbf{a}^{n}, \mathbf{a}^{n+1}\right)=\frac{\Sigma_{i}}{4}\left[a_{i}^{n+1}+a_{i}^{n}\right]\left[\left(a_{j}^{n+1}+a_{k}^{n+1}\right)^{2}+\left(a_{j}^{n}+a_{k}^{n}\right)^{2}\right] \\
& +\frac{\Sigma_{j}}{4}\left[\left(a_{j}^{n+1}\right)^{2}+\left(a_{j}^{n}\right)^{2}\right]\left[a_{i}^{n+1}+a_{k}^{n+1}+a_{i}^{n}+a_{k}^{n}\right] \\
& +\frac{\Sigma_{k}}{4}\left[\left(a_{k}^{n+1}\right)^{2}+\left(a_{k}^{n}\right)^{2}\right]\left[a_{i}^{n+1}+a_{j}^{n+1}+a_{i}^{n}+a_{j}^{n}\right] \\
& +\Lambda\left[a_{i}^{n}+a_{i}^{n+1}\right]\left[\left(a_{j}^{n}\right)^{2}\left(a_{k}^{n}\right)^{2}+\frac{1}{2}\left(a_{j}^{n+1}\right)^{2}\left(a_{k}^{n}\right)^{2}+\frac{1}{2}\left(a_{j}^{n}\right)^{2}\left(a_{k}^{n+1}\right)^{2}+\left(a_{j}^{n+1}\right)^{2}\left(a_{k}^{n+1}\right)^{2}\right] .
\end{aligned}
$$

This scheme was built in order to ensure the following equality:

$$
F\left(\mathbf{a}^{n+1}\right)-F\left(\mathbf{a}^{n}\right)=\sum_{i=1}^{3} d_{i}^{F}\left(\mathbf{a}^{n}, \mathbf{a}^{n+1}\right)\left(a_{i}^{n+1}-a_{i}^{n}\right), \forall\left(\mathbf{a}^{n}, \mathbf{a}^{n+1}\right) \in \mathcal{S}^{2},
$$

and consequently a discrete energy equality which is obtained by multiplying the first equation of the Cahn-Hilliard system by $\mu_{i}^{n+1}$, the second one by $c_{i}^{n+1}-c_{i}^{n}$, writing the equality of left hand sides and summing for $i=1,2,3$.

2.1.2. Navier-Stokes system. We now present the time discretization of the momentum balance of the Navier-Stokes system:

$$
\begin{aligned}
\underbrace{\sqrt{\varrho(\mathbf{c})} \frac{\partial}{\partial t}(\sqrt{\varrho(\mathbf{c})} \mathbf{u})}_{(1)} & +\underbrace{(\varrho(\mathbf{c}) \mathbf{u} \cdot \nabla) \mathbf{u}+\frac{\mathbf{u}}{2} \operatorname{div}(\varrho(\mathbf{c}) \mathbf{u})}_{(2)} \\
& -\underbrace{\operatorname{div}(2 \eta(\mathbf{c}) D(\mathbf{u}))}_{(3)}+\nabla p=\sum_{i=1}^{3} \mu_{i} \nabla c_{i}+\varrho(\mathbf{c}) \mathbf{g} .
\end{aligned}
$$

We separately present the discretization of the different terms (1), (2) and (3) involved in the above equation; for each of them, we give their contribution to the energy balance obtained at the discrete level by multiplying the equation by $\mathbf{u}^{n+1}$. 
Term (1): Using the formal equality $\sqrt{\varrho} \partial_{t}(\sqrt{\varrho} \mathbf{u})=\varrho \partial_{t} \mathbf{u}+\frac{1}{2} \partial_{t} \varrho \mathbf{u}$, we choose the following discretization of term (1) (see [21]):

$$
\varrho^{n} \frac{\mathbf{u}^{n+1}-\mathbf{u}^{n}}{\Delta t}+\frac{1}{2} \frac{\varrho^{n+1}-\varrho^{n}}{\Delta t} \mathbf{u}^{n+1}=\frac{\frac{\varrho^{n}+\varrho^{n+1}}{2} \mathbf{u}^{n+1}-\varrho^{n} \mathbf{u}^{n}}{\Delta t} .
$$

Its contribution to the energy balance is:

$$
\begin{gathered}
\int_{\Omega} \varrho^{n} \frac{\mathbf{u}^{n+1}-\mathbf{u}^{n}}{\Delta t} \cdot \mathbf{u}^{n+1} d x+\int_{\Omega} \frac{1}{2} \frac{\varrho^{n+1}-\varrho^{n}}{\Delta t} \mathbf{u}^{n+1} \cdot \mathbf{u}^{n+1} d x \\
=\frac{1}{2 \Delta t}\left[\left|\sqrt{\varrho^{n+1}} \mathbf{u}^{n+1}\right|_{\mathrm{L}^{2}(\Omega)}^{2}-\left|\sqrt{\varrho^{n}} \mathbf{u}^{n}\right|_{\mathrm{L}^{2}(\Omega)}^{2}+\left|\sqrt{\varrho^{n}}\left(\mathbf{u}^{n+1}-\mathbf{u}^{n}\right)\right|_{\mathrm{L}^{2}(\Omega)}^{2}\right]
\end{gathered}
$$

Term (2): The term (2) is linearized by using an explicit velocity for the transport:

$$
\left(\varrho^{n+1} \mathbf{u}^{n} \cdot \nabla\right) \mathbf{u}^{n+1}+\frac{\mathbf{u}^{n+1}}{2} \operatorname{div}\left(\varrho^{n+1} \mathbf{u}^{n}\right) .
$$

Its contribution to the energy balance vanishes. Indeed, for all $\boldsymbol{\nu}^{\mathbf{u}} \in \mathcal{V}_{0}^{\mathbf{u}}$, we have:

$$
\begin{aligned}
\int_{\Omega}\left(\varrho^{n+1} \mathbf{u}^{n} \cdot \nabla\right) \mathbf{u}^{n+1} \cdot \boldsymbol{\nu}^{\mathbf{u}} d x+\int_{\Omega} \frac{1}{2} \operatorname{div}\left(\varrho^{n+1} \mathbf{u}^{n}\right) \mathbf{u}^{n+1} \cdot \boldsymbol{\nu}^{\mathbf{u}} d x \\
=\frac{1}{2}\left[\int_{\Omega}\left(\varrho^{n+1} \mathbf{u}^{n} \cdot \nabla\right) \mathbf{u}^{n+1} \cdot \boldsymbol{\nu}^{\mathbf{u}} d x-\int_{\Omega}\left(\varrho^{n+1} \mathbf{u}^{n} \cdot \nabla\right) \boldsymbol{\nu}^{\mathbf{u}} \cdot \mathbf{u}^{n+1} d x\right]
\end{aligned}
$$

In particular, when we take $\boldsymbol{\nu}^{\mathbf{u}}=\mathbf{u}^{n+1}$, the above term vanishes.

Term (3): We discretize the term (3) with an implicit scheme: $-\operatorname{div}\left(2 \eta^{n+1} D \mathbf{u}^{n+1}\right)$. Its contribution to the energy balance is: $\int_{\Omega} \eta^{n+1}\left|D \mathbf{u}^{n+1}\right|^{2} d x$.

Thus, we adopt the following discretization of the Navier-Stokes equation:

$$
\left\{\begin{array}{c}
\varrho^{n} \frac{\mathbf{u}^{n+1}-\mathbf{u}^{n}}{\Delta t}+\frac{1}{2} \frac{\varrho^{n+1}-\varrho^{n}}{\Delta t} \mathbf{u}^{n+1}+\left(\varrho^{n+1} \mathbf{u}^{n} \cdot \nabla\right) \mathbf{u}^{n+1}+\frac{\mathbf{u}^{n+1}}{2} \operatorname{div}\left(\varrho^{n+1} \mathbf{u}^{n}\right) \\
-\operatorname{div}\left(\eta^{n+1} D \mathbf{u}^{n+1}\right)+\nabla p^{n+1}=\underset{\text { capillary }}{\text { forces term }}+\varrho^{n+1} \mathbf{g}, \\
\operatorname{div}\left(\mathbf{u}^{n+1}\right)=0 .
\end{array}\right.
$$

The discretization of the capillary forces term is described in the next paragraph.

2.1.3. Coupling terms. We give in this paragraph the discretization of coupling terms. That is the transport terms $\mathbf{u} \cdot \nabla c_{i}$ in the Cahn-Hilliard equations, and the capillary forces term $\sum_{i=1}^{3} \mu_{i} \nabla c_{i}$ in the momentum balance (Navier-Stokes equation). At the continuous level, when writing the energy balance, the contributions of these two terms counterbalance each other. At the discrete level, we saw that the energy balance is obtained, for the Cahn-Hilliard system, by multiplying the transport terms by $\mu_{i}^{n+1}$ before summing up for $i=1,2,3$ and, for the Navier-Stokes equation, by multiplying the capillary forces term by $\mathbf{u}^{n+1}$.

Consequently, it is easy to see that when all the terms mentioned above are discretized with an implicit scheme ( $c f[12]$ for the diphasic case), i.e. $\mathbf{u}^{n+1} \cdot \nabla c_{i}^{n+1}$ and $\sum_{i=1}^{3} \mu_{i}^{n+1} \nabla c_{i}^{n+1}$, the balance is also true at the discrete level. However, this 
discretization introduces a strong coupling between the Cahn-Hilliard and NavierStokes systems. The discrete system is difficult to solve in practice.

It is possible to uncouple the system ( $c f$ [18] for the diphasic case, [6] for the triphasic case) by using an explicit velocity (i.e. the velocity at time $t^{n}$ ) in the Cahn-Hilliard equation: $\mathbf{u}^{n} \cdot \nabla c_{i}^{n+1}$. However, the contributions of the transport terms in the Cahn-Hilliard system and the contribution of the capillary forces in the Navier-Stokes equation do not counterbalance when writing the discrete energy balance which contains the additional term: $\left(\mathbf{u}^{n+1}-\mathbf{u}^{n}\right) \cdot \sum_{i=1}^{3} \mu_{i}^{n+1} \nabla c_{i}^{n+1}$. It is difficult to attribute a sign to this term and the scheme stability is obtained only conditionally ( $c f[18]$, assuming for instance that the ratio between the time step and the mesh size is bounded).

We first observe that it is possible to uncouple the resolution of the Navier-Stokes system and the taking into account of capillary forces. The taking into account of the capillary forces is performed during a first step which provides an intermediate velocity $\mathbf{u}^{*}$ which is then used in the Cahn-Hilliard system. The Navier-Stokes system is then solved in a second step. The scheme reads:

(i) Taking into account of capillary forces:

$$
\left\{\begin{array}{l}
\varrho^{n} \frac{\mathbf{u}^{*}-\mathbf{u}^{n}}{\Delta t}+\nabla p^{*}=\sum_{i=1}^{3} \mu_{i}^{n+1} \nabla c_{i}^{n+1} \\
\operatorname{div}\left(\mathbf{u}^{*}\right)=0
\end{array}\right.
$$

(ii) Cahn-Hilliard system:

$$
\left\{\begin{array}{l}
\frac{c_{i}^{n+1}-c_{i}^{n}}{\Delta t}+\mathbf{u}^{*} \cdot \nabla c_{i}^{n+1}=\operatorname{div}\left(\frac{M_{0}^{n+\alpha}}{\Sigma_{i}} \nabla \mu_{i}^{n+1}\right) \\
\mu_{i}^{n+1}=D_{i}^{F}\left(\mathbf{c}^{n}, \mathbf{c}^{n+1}\right)-\frac{3}{4} \varepsilon \Sigma_{i} \Delta c_{i}^{n+\beta}
\end{array}\right.
$$

(iii) Navier-Stokes system:

$$
\left\{\begin{array}{l}
\varrho^{n} \frac{\mathbf{u}^{n+1}-\mathbf{u}^{*}}{\Delta t}+\frac{1}{2} \frac{\varrho^{n+1}-\varrho^{n}}{\Delta t} \mathbf{u}^{n+1}+\left(\varrho^{n+1} \mathbf{u}^{n} \cdot \nabla\right) \mathbf{u}^{n+1}+\frac{\mathbf{u}^{n+1}}{2} \operatorname{div}\left(\varrho^{n+1} \mathbf{u}^{n}\right) \\
-\operatorname{div}\left(\eta^{n+1} D \mathbf{u}^{n+1}\right)+\nabla\left(p^{n+1}-p^{*}\right)=\varrho^{n+1} \mathbf{g} \\
\operatorname{div}\left(\mathbf{u}^{n+1}\right)=0 .
\end{array}\right.
$$

This discretization is unconditionally stable but the system of step (i) (Darcy problem) is still coupled with the Cahn-Hilliard equations (system (ii)).

We propose to forget for a moment the divergence free constraint imposed to $\mathbf{u}^{*}$ (and consequently the associated pressure term $\nabla p^{*}$ ) in the system of the step (i). This leads to define $\mathbf{u}^{*}$ as follows:

$$
\varrho^{n} \frac{\mathbf{u}^{*}-\mathbf{u}^{n}}{\Delta t}=\sum_{i=1}^{3} c_{i}^{n+1} \nabla \mu_{i}^{n+1} .
$$

This definition of $\mathbf{u}^{*}$ is explicit and $\mathbf{u}^{*}$ can be replaced by its expression in the CahnHilliard system thus eliminating the coupling with the Navier-Stokes equation.

The problem is that $\mathbf{u}^{*}$ is not divergence free. Nevertheless, note that the divergence of $\mathbf{u}^{*}$ is of order $O(\Delta t)$ and that the property $\mathbf{u}^{*} \cdot \mathbf{n}=0$ is still satisfied on 
$\Gamma$. Now, the question is: is it possible to discretize the transport term in the CahnHilliard equation in order to preserve its fundamental properties (volume conservation and the fact that the sum of the three order parameters remains equal to 1) ? An answer is given in the next paragraph.

2.1.4. Transport term in the Cahn-Hilliard system when the velocity is not divergence free. In this paragraph, we are interested in the form of the transport term in the Cahn-Hilliard equation when the advection velocity, denoted by $\mathbf{u}^{*}$ is not divergence free but satisfy the boundary condition $\mathbf{u}^{*} \cdot \mathbf{n}=0$ on $\Gamma$.

Preserving properties of the Cahn-Hilliard when the advective velocity is not divergence free may be useful in other contexts. For instance, when using an incremental projection method ( $c f[9],[26]$ ), the end step velocity is not divergence free.

The transport term may be written in the conservative or non conservative form (these two forms are not equivalent since a priori $\operatorname{div}\left(\mathbf{u}^{*}\right) \neq 0$ ):

- non conservative form: $\mathbf{u}^{*} \cdot \nabla c_{i}$,

- conservative form: $\operatorname{div}\left(c_{i} \mathbf{u}^{*}\right)$.

The conservative form ensures the total volume conservation of each phase (since $\mathbf{u}^{*} \cdot \mathbf{n}=0$ on $\Gamma$ ). This is not the case for the non conservative form since a priori $\int_{\Omega} \mathbf{u}^{*} \cdot \nabla c_{i} d x \neq 0$. Conversely, when using the conservative form, a necessary condition to ensure that the sum of the three order parameters $c_{i}$ remains constant equal to 1 , is $\operatorname{div}\left(\mathbf{u}^{*}\right)=0$. Neither the conservative form nor the non conservative form ensures both volume conservation and the fact that the sum of the three order parameters remains equal to 1 .

We propose to use the following formulation:

$$
\operatorname{div}\left(\left(c_{i}-\alpha_{i}\right) \mathbf{u}^{*}\right)
$$

where $\alpha_{i}$ is a constant. This formulation allows to ensure the two desired properties if $\sum_{i=1}^{3} \alpha_{i}=1$. To guarantee the consistency with diphasic model, the constant $\alpha_{i}$ may be zero when the phase $i$ is not present. In the sequel, we propose to choose:

$$
\alpha_{i}=\int_{\Omega} c_{i}^{0} d x .
$$

This formulation allows to use an advective velocity which is not divergence free. The term $-\alpha_{i} \operatorname{div}\left(\mathbf{u}^{*}\right)$ is added in the Cahn-Hilliard system, its role is to re-equilibrate the values of each order parameters to ensure the fact that their sum remains equal to 1 . We prove in section 5 that this term is of order $O(h+\Delta t)$ and so it does not disturb the consistency of the scheme.

Owing to this formulation of the transport term, it seems natural to adopt the following definition for the capillary forces term in the Navier-Stokes equation:

$$
-\sum_{i=1}^{3}\left(c_{i}-\alpha_{i}\right) \nabla \mu_{i} .
$$

This is equivalent to modify the definition of the pressure by subtracting the term $\sum_{i=1}^{3}\left(c_{i}-\alpha_{i}\right) \mu_{i}$.

2.1.5. Time discretization of the Cahn-Hilliard/Navier-Stokes system. Finally, the different considerations presented in the previous paragraphs lead to propose the following scheme:

PROBLEM 1. 
- Step 1: resolution of the Cahn-Hilliard system

Find $\left(\mathbf{c}^{n+1}, \boldsymbol{\mu}^{n+1}\right) \in\left(\mathcal{V}^{c}\right)^{3} \times\left(\mathcal{V}^{\mu}\right)^{3}$ such that, for $i=1,2$ and 3 ,

$$
\left\{\begin{array}{r}
\frac{c_{i}^{n+1}-c_{i}^{n}}{\Delta t}+\operatorname{div}\left(\left[c_{i}^{n}-\alpha_{i}\right]\left[\mathbf{u}^{n}-\frac{\Delta t}{\varrho^{n}} \sum_{j=1}^{3}\left(c_{j}^{n}-\alpha_{j}\right) \nabla \mu_{j}^{n+1}\right]\right) \\
=\operatorname{div}\left(\frac{M_{0}^{n+\alpha}}{\Sigma_{i}} \nabla \mu_{i}^{n+1}\right), \\
\mu_{i}^{n+1}=D_{i}^{F}\left(\mathbf{c}^{n}, \mathbf{c}^{n+1}\right)-\frac{3}{4} \Sigma_{i} \varepsilon \Delta c_{i}^{n+\beta},
\end{array}\right.
$$

with $\alpha_{j}$ a constant: $\alpha_{j}=\int_{\Omega} c_{j}^{0} d x$.

- Step 2: resolution of the Navier-Stokes system

Find $\left(\mathbf{u}^{n+1}, p^{n+1}\right) \in \mathcal{V}_{0}^{\mathbf{u}} \times \mathcal{V}^{p}$ such that,

$$
\left\{\begin{array}{c}
\varrho^{n} \frac{\mathbf{u}^{n+1}-\mathbf{u}^{n}}{\Delta t}+\frac{1}{2} \frac{\varrho^{n+1}-\varrho^{n}}{\Delta t} \mathbf{u}^{n+1}+\left(\varrho^{n+1} \mathbf{u}^{n} \cdot \nabla\right) \mathbf{u}^{n+1} \\
+\frac{\mathbf{u}^{n+1}}{2} \operatorname{div}\left(\varrho^{n+1} \mathbf{u}^{n}\right)+\operatorname{div}\left(2 \eta^{n+1} D \mathbf{u}^{n+1}\right) \\
\quad+\nabla p^{n+1}=\varrho^{n+1} \mathbf{g}+\sum_{j=1}^{3}\left(c_{j}^{n}-\alpha_{j}\right) \nabla \mu_{j}^{n+1}, \\
\operatorname{div}\left(\mathbf{u}^{n+1}\right)=0,
\end{array}\right.
$$

where $\eta^{n+1}=\eta\left(\mathbf{c}_{h}^{n+1}\right)$ and $\varrho^{\ell}=\varrho\left(\mathbf{c}^{\ell}\right)$, for $\ell=n$ and $\ell=n+1$.

2.2. Space discretization. For the space discretization, we use the Galerkin method and the finite elements method. Let $\mathcal{V}_{h}^{c}, \mathcal{V}_{h}^{\mu}, \mathcal{V}_{h}^{\mathbf{u}}$ and $\mathcal{V}_{h}^{p}$ be finite elements approximation spaces of $\mathcal{V}^{c}, \mathcal{V}^{\mu}, \mathcal{V}^{\mathbf{u}}$ and $\mathcal{V}^{p}$ respectively. Since the velocity satisfies homogeneous Dirichlet boundary conditions on $\Gamma$, we define the following approximation space:

$$
\mathcal{V}_{h, 0}^{\mathbf{u}}=\left\{\nu_{h}^{\mathbf{u}} \in \mathcal{V}_{h}^{\mathbf{u}} ; \nu_{h}^{\mathbf{u}}=0 \text { on } \Gamma\right\} .
$$

To simplify the notation, we introduce also the following space:

$$
\mathcal{V}_{h, \mathcal{S}}^{\mathbf{c}}=\left\{\mathbf{c}_{h}=\left(c_{1 h}, c_{2 h}, c_{3 h}\right) \in\left(\mathcal{V}_{h}^{c}\right)^{3} ; \mathbf{c}_{h}(x) \in \mathcal{S} \text { for almost every } x \in \Omega\right\} .
$$

We require some standard assumptions on approximation spaces:

$$
\begin{aligned}
& \forall\left(\nu^{c}, \nu^{\mu}\right) \in \mathcal{V}^{c} \times \mathcal{V}^{\mu}, \inf _{\nu_{h}^{c} \in \mathcal{V}_{h}^{c}}\left|\nu^{c}-\nu_{h}^{c}\right|_{\mathrm{H}^{1}(\Omega)}+\inf _{\nu_{h}^{\mu} \in \mathcal{V}_{h}^{\mu}}\left|\nu^{\mu}-\nu_{h}^{\mu}\right|_{\mathrm{H}^{1}(\Omega)} \underset{h \rightarrow 0}{\longrightarrow} 0, \\
& \forall\left(\boldsymbol{\nu}^{\mathbf{u}}, \nu^{p}\right) \in \mathcal{V}_{0}^{\mathbf{u}} \times \mathcal{V}^{p}, \inf _{\boldsymbol{\nu}_{h}^{\mathbf{u}} \in \mathcal{V}_{h, 0}^{\mathbf{u}}}\left|\boldsymbol{\nu}^{\mathbf{u}}-\boldsymbol{\nu}_{h}^{\mathbf{u}}\right|_{\left(\mathrm{H}^{1}(\Omega)\right)^{d}}+\inf _{\nu_{h}^{p} \in \mathcal{V}_{h}^{p}}\left|\nu^{p}-\nu_{h}^{p}\right|_{\left(\mathrm{L}^{2}(\Omega)\right)^{d}} \underset{h \rightarrow 0}{\longrightarrow} 0,
\end{aligned}
$$

Moreover, we assume that $1 \in \mathcal{V}_{h}^{c}, \mathcal{V}_{h}^{c} \subset \mathcal{V}_{h}^{\mu}$ and that the $L^{2}$-projection $\Pi_{0}^{\mathcal{V}_{h}^{c}}\left(\right.$ resp. $\Pi_{0}^{\mathcal{V}_{h}^{\mu}}$ ) on the approximation space $\mathcal{V}_{h}^{c}\left(\right.$ resp. $\left.\mathcal{V}_{h}^{\mu}\right)$ is stable in $\mathrm{H}^{1}$, i.e. there exists a positive constant $C$ independent of $h$ such that:

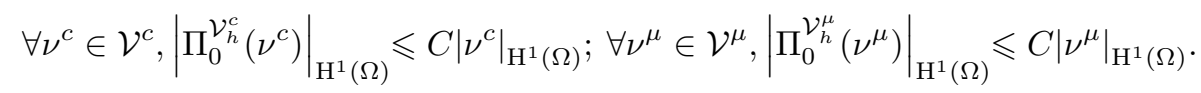


We assume that the approximation space for order parameters satisfies an inverse inequality: there exists a function $\mathrm{C}_{\mathrm{inv}}$ of $h$ such that

$$
\forall \nu_{h}^{c} \in \mathcal{V}_{h}^{c}, \quad\left|\nu_{h}^{c}\right|_{\mathrm{L}^{\infty}(\Omega)}^{2} \leqslant \mathrm{C}_{\mathrm{inv}}(h)\left|\nu_{h}^{c}\right|_{\mathrm{H}^{1}(\Omega)}^{2}
$$

This property is (for instance) satisfied when the mesh family is quasi-uniform and the approximation spaces are associated to corresponding Lagrange finite elements; in this case we can choose $\mathrm{C}_{\mathrm{inv}}(h)=\mathrm{C}(1+|\ln (h)|)$ for $d=2$ and $\mathrm{C}_{\mathrm{inv}}(h)=\mathrm{C}^{-1}$ for $d=3$ where $\mathrm{C}$ is a constant which only depends on the mesh regularity $(c f[8,4.5 .11$ (p. 112) and 4.9.2 (p. 123)]).

Finally, we assume that the approximation spaces for velocity and pressure satisfy the so-called uniform inf-sup condition: there exists a positive constant $\Theta$ (independent of $h$ ) such that

$$
\inf _{\nu_{h}^{p} \in \mathcal{V}_{h}^{p}} \sup _{\boldsymbol{\nu}_{h}^{\mathbf{u}} \in \mathcal{V}_{h, 0}^{\mathbf{u}}} \frac{\int_{\Omega} \nu_{h}^{p} \operatorname{div} \boldsymbol{\nu}_{h}^{\mathbf{u}} d x}{\left|\nu_{h}^{p}\right|_{\mathrm{L}^{2}(\Omega)}\left|\boldsymbol{\nu}_{h}^{\mathbf{u}}\right|_{\left(\mathrm{H}^{1}(\Omega)\right)^{d}}} \geqslant \Theta .
$$

We begin with the definition of discrete functions $\mathbf{c}_{h}^{0} \in \mathcal{V}_{h, \mathcal{S}}^{\mathbf{c}}$ and $\mathbf{u}_{h}^{0} \in \mathcal{V}_{h, 0}^{\mathbf{u}}$ at the initial time satisfying:

$$
\left|\mathbf{c}_{h}^{0}-\mathbf{c}^{0}\right|_{\left(\mathrm{H}^{1}(\Omega)\right)^{3}} \underset{h \rightarrow 0}{\longrightarrow} 0 \quad \text { and } \quad\left|\mathbf{u}_{h}^{0}-\mathbf{u}^{0}\right|_{\left(\mathrm{H}^{1}(\Omega)\right)^{d}} \underset{h \rightarrow 0}{\longrightarrow} 0
$$

These discrete functions $\mathbf{c}_{h}^{0}$ and $\mathbf{u}_{h}^{0}$ can be obtained from initial conditions $\mathbf{c}^{0}$ and $\mathbf{u}^{0}$ by $\mathrm{H}^{1}(\Omega)$ projection, or as it is the case in practice, by finite elements interpolation provided that $c_{i}^{0}$ and $\mathbf{u}^{0}$ are smooth enough.

Assume now that $\mathbf{c}_{h}^{n} \in \mathcal{V}_{h, \mathcal{S}}^{\mathrm{c}}$ and $\mathbf{u}_{h}^{n} \in \mathcal{V}_{h, 0}^{\mathrm{u}}$ are given, the Galerkin approximation of problem 1 reads:

Problem 2 (Formulation with three order parameters).

Step 1: resolution of the Cahn-Hilliard system

Find $\left(\mathbf{c}_{h}^{n+1}, \boldsymbol{\mu}_{h}^{n+1}\right) \in\left(\mathcal{V}_{h, \mathcal{S}}^{\mathbf{c}}\right)^{3} \times\left(\mathcal{V}_{h}^{\mu}\right)^{3}$ s. $t . \forall \nu_{h}^{c} \in \mathcal{V}_{h}^{c}, \forall \nu_{h}^{\mu} \in \mathcal{V}_{h}^{\mu}$, for $i=1,2,3$,

$$
\left\{\begin{aligned}
& \int_{\Omega} \frac{c_{i h}^{n+1}-c_{i h}^{n}}{\Delta t} \nu_{h}^{\mu} d x-\int_{\Omega}\left[c_{i h}^{n}-\alpha_{i h}\right]\left[\mathbf{u}_{h}^{n}-\frac{\Delta t}{\varrho_{h}^{n}} \sum_{j=1}^{3}\left(c_{j h}^{n}-\alpha_{j h}\right) \nabla \mu_{j h}^{n+1}\right] \cdot \nabla \nu_{h}^{\mu} d x \\
&=-\int_{\Omega} \frac{M_{0 h}^{n+\alpha}}{\Sigma_{i}} \nabla \mu_{i h}^{n+1} \cdot \nabla \nu_{h}^{\mu} d x \\
& \int_{\Omega} \mu_{i h}^{n+1} \nu_{h}^{c} d x=\int_{\Omega} D_{i}^{F}\left(\mathbf{c}_{h}^{n}, \mathbf{c}_{h}^{n+1}\right) \nu_{h}^{c} d x+\int_{\Omega} \frac{3}{4} \Sigma_{i} \varepsilon \nabla c_{i h}^{n+\beta} \nabla \nu_{h}^{c} d x,
\end{aligned}\right.
$$

where $\alpha_{j h}$ is the constant defined by $\alpha_{j h}=\int_{\Omega} c_{j h}^{0} d x$.

Step 2: resolution of the Navier-Stokes equation 
Find $\left(\mathbf{u}_{h}^{n+1}, p_{h}^{n+1}\right) \in \mathcal{V}_{h, 0}^{\mathbf{u}} \times \mathcal{V}_{h}^{p}$ such that $\forall \boldsymbol{\nu}_{h}^{\mathbf{u}} \in \mathcal{V}_{h, 0}^{\mathbf{u}}, \forall \nu_{h}^{p} \in \mathcal{V}_{h}^{p}$,

$$
\left\{\begin{array}{c}
\int_{\Omega} \varrho_{h}^{n} \frac{\mathbf{u}_{h}^{n+1}-\mathbf{u}_{h}^{n}}{\Delta t} \boldsymbol{\nu}_{h}^{\mathbf{u}} d x+\frac{1}{2} \int_{\Omega} \frac{\varrho_{h}^{n+1}-\varrho_{h}^{n}}{\Delta t} \mathbf{u}_{h}^{n+1} \cdot \boldsymbol{\nu}_{h}^{\mathbf{u}} d x \\
+\frac{1}{2} \int_{\Omega} \varrho_{h}^{n+1}\left(\mathbf{u}_{h}^{n} \cdot \nabla\right) \mathbf{u}_{h}^{n+1} \cdot \boldsymbol{\nu}_{h}^{\mathbf{u}} d x-\frac{1}{2} \int_{\Omega} \varrho_{h}^{n+1}\left(\mathbf{u}_{h}^{n} \cdot \nabla\right) \boldsymbol{\nu}_{h}^{\mathbf{u}} \cdot \mathbf{u}_{h}^{n+1} d x \\
+\int_{\Omega} 2 \eta_{h}^{n+1} D \mathbf{u}_{h}^{n+1}: D \boldsymbol{\nu}_{h}^{\mathbf{u}} d x-\int_{\Omega} p_{h}^{n+1} \operatorname{div}\left(\boldsymbol{\nu}_{h}^{\mathbf{u}}\right) d x \\
=\int_{\Omega} \varrho_{h}^{n+1} \mathbf{g} \cdot \boldsymbol{\nu}_{h}^{\mathbf{u}} d x-\int_{\Omega} \sum_{j=1}^{3}\left(c_{j h}^{n}-\alpha_{j h}\right) \nabla \mu_{j h}^{n+1} \cdot \boldsymbol{\nu}_{h}^{\mathbf{u}} d x \\
\int_{\Omega} \nu_{h}^{p} \operatorname{div}\left(\mathbf{u}_{h}^{n+1}\right) d x=0
\end{array}\right.
$$

where $\eta_{h}^{n+1}=\eta\left(\mathbf{c}_{h}^{n+1}\right)$ and $\varrho_{h}^{\ell}=\varrho\left(\mathbf{c}_{h}^{\ell}\right)$, for $\ell=n$ and $\ell=n+1$.

REMARK 2. For the resolution of the Cahn-Hilliard system, it is equivalent to only solve the equations satisfied by $\left(c_{1 h}^{n+1}, c_{2 h}^{n+1}, \mu_{1 h}^{n+1}, \mu_{2 h}^{n+1}\right)$ and then to deduce the unknowns $\left(c_{3 h}^{n+1}, \mu_{3 h}^{n+1}\right)$ using the following relationships:

$$
c_{3 h}^{n+1}=1-c_{1 h}^{n+1}-c_{2 h}^{n+1} \quad \text { and } \quad \mu_{3 h}^{n+1}=-\left(\frac{\Sigma_{3}}{\Sigma_{1}} \mu_{1 h}^{n+1}+\frac{\Sigma_{3}}{\Sigma_{2}} \mu_{2 h}^{n+1}\right) .
$$

The resolution of the Navier-Stokes system remains unchanged (cf problem 2). In the sequel, in systems where only the unknowns $\left(c_{1 h}^{n+1}, \mu_{1 h}^{n+1}, c_{2 h}^{n+1}, \mu_{2 h}^{n+1}\right)$ are present, the notation $\mathbf{c}_{h}^{n+1}$ stands for the vector $\left(c_{1 h}^{n+1}, c_{2 h}^{n+1}, 1-c_{1 h}^{n+1}-c_{2 h}^{n+1}\right)$.

3. Unconditional stability of the scheme. We prove in this section the energy equality which ensures the unconditional stability of the scheme.

Proposition 3.1 (Discrete energy equality). Let $\mathbf{c}_{h}^{n} \in \mathcal{V}_{h, \mathcal{S}}^{\mathbf{c}}$ and $\mathbf{u}_{h}^{n} \in \mathcal{V}_{h, 0}^{\mathbf{u}}$. Assume that there exists a solution $\left(\mathbf{c}_{h}^{n+1}, \boldsymbol{\mu}_{h}^{n+1}, \mathbf{u}_{h}^{n+1}, p_{h}^{n+1}\right)$ of problem 2. Then, we have the following equality:

$$
\begin{aligned}
& {\left[\mathcal{F}_{\mathbf{\Sigma}, \varepsilon}^{\text {triph }}\left(\mathbf{c}_{h}^{n+1}\right)+\frac{1}{2} \int_{\Omega} \varrho_{h}^{n+1}\left|\mathbf{u}_{h}^{n+1}\right|^{2} d x\right]-\left[\mathcal{F}_{\mathbf{\Sigma}, \varepsilon}^{\text {triph }}\left(\mathbf{c}_{h}^{n}\right)+\frac{1}{2} \int_{\Omega} \varrho_{h}^{n}\left|\mathbf{u}_{h}^{n}\right|^{2} d x\right]} \\
& +\Delta t \sum_{i=1}^{3} \int_{\Omega} \frac{M_{0 h}^{n+\alpha}}{\Sigma_{i}}\left|\nabla \mu_{i h}^{n+1}\right|^{2} d x+\Delta t \int_{\Omega} 2 \eta_{h}^{n+1}\left|D \mathbf{u}_{h}^{n+1}\right|^{2} d x \\
& +\frac{3}{8}(2 \beta-1) \varepsilon \int_{\Omega} \sum_{i=1}^{3} \Sigma_{i}\left|\nabla c_{i h}^{n+1}-\nabla c_{i h}^{n}\right|^{2} d x+\frac{1}{2} \int_{\Omega} \varrho_{h}^{n}\left[\left|\mathbf{u}_{h}^{n+1}-\mathbf{u}^{*}\right|^{2}+\left|\mathbf{u}^{*}-\mathbf{u}_{h}^{n}\right|^{2}\right] d x \\
& =\frac{12}{\varepsilon} \int_{\Omega}\left[F\left(\mathbf{c}_{h}^{n+1}\right)-F\left(\mathbf{c}_{h}^{n}\right)-\mathbf{d}^{F}\left(\mathbf{c}_{h}^{n}, \mathbf{c}_{h}^{n+1}\right) \cdot\left(\mathbf{c}_{h}^{n+1}-\mathbf{c}_{h}^{n}\right)\right] d x+\Delta t \int_{\Omega} \varrho_{h}^{n+1} \mathbf{g} \cdot \mathbf{u}_{h}^{n+1} d x,
\end{aligned}
$$

where $\mathbf{d}^{F}(\cdot, \cdot)$ is the vector $\left(d_{i}^{F}(\cdot, \cdot)\right)_{i=1,2,3}$ and

$$
\mathbf{u}^{*}=\mathbf{u}_{h}^{n}-\frac{\Delta t}{\varrho_{h}^{n}} \sum_{j=1}^{3}\left(c_{j h}^{n}-\alpha_{j}\right) \nabla \mu_{j h}^{n+1} .
$$

Proof. The key point of the proof is the following observation: the Cahn-Hilliard and Navier-Stokes systems can be re-written using the function $\mathbf{u}^{*}$ defined by (3.2). Then, standard estimations for the Cahn-Hilliard and Navier-Stokes systems are done (step 1 and 3) and an estimation on the $\mathrm{L}^{2}$ norm of $\mathbf{u}^{*}$ gives the conclusion (step 2). 
Step 1: Owing to the definition (3.2) of the function $\mathbf{u}^{*}$, we observe that the system 2.3 can be rewritten as follows:

$$
\left\{\begin{array}{l}
\int_{\Omega} \frac{c_{i h}^{n+1}-c_{i h}^{n}}{\Delta t} \nu_{h}^{\mu} d x-\int_{\Omega}\left[c_{i h}^{n}-\alpha_{i}\right] \mathbf{u}^{*} \cdot \nabla \nu_{h}^{\mu} d x=-\int_{\Omega} \frac{M_{0 h}^{n+\alpha}}{\Sigma_{i}} \nabla \mu_{i h}^{n+1} \cdot \nabla \nu_{h}^{\mu} d x \\
\int_{\Omega} \mu_{i h}^{n+1} \nu_{h}^{c} d x=\int_{\Omega} D_{i}^{F}\left(\mathbf{c}_{h}^{n}, \mathbf{c}_{h}^{n+1}\right) \nu_{h}^{c} d x+\int_{\Omega} \frac{3}{4} \Sigma_{i} \varepsilon \nabla c_{i h}^{n+\beta} \nabla \nu_{h}^{c} d x
\end{array}\right.
$$

We take $\nu_{h}^{\mu}=\mu_{i h}^{n+1}$ and $\nu_{h}^{c}=\frac{c_{i h}^{n+1}-c_{i h}^{n}}{\Delta t}$ as test functions in this system. After some standard calculation (see [7]), this yields:

$$
\begin{aligned}
\mathcal{F}_{\boldsymbol{\Sigma}, \varepsilon}^{\mathrm{triph}}\left(\mathbf{c}_{h}^{n+1}\right)-\mathcal{F}_{\boldsymbol{\Sigma}, \varepsilon}^{\mathrm{triph}}\left(\mathbf{c}_{h}^{n}\right)+\Delta t \sum_{i=1}^{3} \int_{\Omega} \frac{M_{0 h}^{n+\alpha}}{\Sigma_{i}}\left|\nabla \mu_{i h}^{n+1}\right|^{2} d x \\
+\frac{3}{8}(2 \beta-1) \varepsilon \int_{\Omega} \sum_{i=1}^{3} \Sigma_{i}\left|\nabla c_{i h}^{n+1}-\nabla c_{i h}^{n}\right|^{2} d x=\Delta t \int_{\Omega} \mathbf{u}^{*} \cdot \sum_{i=1}^{3}\left(c_{i h}^{n}-\alpha_{i}\right) \nabla \mu_{i h}^{n+1} d x \\
+\frac{12}{\varepsilon} \int_{\Omega}\left[F\left(\mathbf{c}_{h}^{n+1}\right)-F\left(\mathbf{c}_{h}^{n}\right)-\mathbf{d}^{F}\left(\mathbf{c}_{h}^{n}, \mathbf{c}_{h}^{n+1}\right) \cdot\left(\mathbf{c}_{h}^{n+1}-\mathbf{c}_{h}^{n}\right)\right] d x .
\end{aligned}
$$

Step 2: It is possible to obtain an estimation of the first term of the right hand side of the previous equality. By definition of $\mathbf{u}^{*}$, we have: $\sqrt{\varrho_{h}^{n}} \mathbf{u}^{*}=\sqrt{\varrho_{h}^{n}} \mathbf{u}_{h}^{n}-$ $\frac{\Delta t}{\sqrt{\varrho_{h}^{n}}} \sum_{j=1}^{3}\left(c_{j h}^{n}-\alpha_{j}\right) \nabla \mu_{j h}^{n+1}$. Multiplying by $\sqrt{\varrho_{h}^{n}} \mathbf{u}^{*}$, and integrating on $\Omega$, yields:

$$
\begin{aligned}
\frac{1}{2} \int_{\Omega} \varrho_{h}^{n}\left|\mathbf{u}^{*}\right|^{2} d x-\frac{1}{2} \int_{\Omega} \varrho_{h}^{n}\left|\mathbf{u}_{h}^{n}\right|^{2} d x & +\frac{1}{2} \int_{\Omega} \varrho_{h}^{n}\left|\mathbf{u}^{*}-\mathbf{u}_{h}^{n}\right|^{2} d x \\
& =-\Delta t \int_{\Omega} \mathbf{u}^{*} \cdot \sum_{j=1}^{3}\left(c_{j h}^{n}-\alpha_{j}\right) \nabla \mu_{j h}^{n+1} d x .
\end{aligned}
$$

Step 3: The system (2.4) can also be re-written using the function $\mathbf{u}^{*}$ :

$$
\left\{\begin{array}{l}
\int_{\Omega} \varrho_{h}^{n} \frac{\mathbf{u}_{h}^{n+1}-\mathbf{u}^{*}}{\Delta t} \boldsymbol{\nu}_{h}^{\mathbf{u}} d x+\frac{1}{2} \int_{\Omega} \mathbf{u}_{h}^{n+1} \frac{\varrho_{h}^{n+1}-\varrho_{h}^{n}}{\Delta t} \boldsymbol{\nu}_{h}^{\mathbf{u}} d x \\
\quad+\frac{1}{2} \int_{\Omega} \varrho_{h}^{n+1}\left(\mathbf{u}_{h}^{n} \cdot \nabla\right) \mathbf{u}_{h}^{n+1} \cdot \boldsymbol{\nu}_{h}^{\mathbf{u}} d x-\frac{1}{2} \int_{\Omega} \varrho_{h}^{n+1}\left(\mathbf{u}_{h}^{n} \cdot \nabla\right) \boldsymbol{\nu}_{h}^{\mathbf{u}} \cdot \mathbf{u}_{h}^{n+1} d x \\
\quad+\int_{\Omega} 2 \eta_{h}^{n+1} D \mathbf{u}_{h}^{n+1}: D \boldsymbol{\nu}_{h}^{\mathbf{u}} d x-\int_{\Omega} p_{h}^{n+1} \operatorname{div}\left(\boldsymbol{\nu}_{h}^{\mathbf{u}}\right) d x=\int_{\Omega} \varrho_{h}^{n+1} \mathbf{g} \cdot \boldsymbol{\nu}_{h}^{\mathbf{u}} d x \\
\int_{\Omega} \nu_{h}^{p} \operatorname{div}\left(\mathbf{u}_{h}^{n+1}\right) d x=0 .
\end{array}\right.
$$

We take $\boldsymbol{\nu}_{h}^{\mathbf{u}}=\mathbf{u}_{h}^{n+1}$ and $\nu_{h}^{p}=p_{h}^{n+1}$ as test functions in this system. This yields:

$$
\begin{aligned}
\frac{1}{2} \int_{\Omega} \varrho_{h}^{n+1}\left|\mathbf{u}_{h}^{n+1}\right|^{2} d & -\frac{1}{2} \int_{\Omega} \varrho_{h}^{n}\left|\mathbf{u}^{*}\right|^{2} d x+\frac{1}{2} \int_{\Omega} \varrho_{h}^{n}\left|\mathbf{u}_{h}^{n+1}-\mathbf{u}^{*}\right|^{2} d x \\
& +\Delta t \int_{\Omega} 2 \eta_{h}^{n+1}\left|D \mathbf{u}_{h}^{n+1}\right|^{2} d x=\Delta t \int_{\Omega} \varrho_{h}^{n+1} \mathbf{g} \cdot \mathbf{u}_{h}^{n+1} d x
\end{aligned}
$$

The conclusion is obtained by summing up the equations (3.3), (3.4) and (3.5). 
REMARK 3. An important difference with the work presented in [18] in the case of a homogeneous diphasic Cahn-Hilliard/Navier-Stokes model is that no condition is required on the time step to ensure the stability.

The previous stability result enables to prove the existence of solutions for the non linear approximate problem 2.

TheOrem 3.2. Given $\mathbf{c}_{h}^{n} \in \mathcal{V}_{h, \mathcal{S}}^{\mathbf{c}}, \mathbf{u}_{h}^{n} \in \mathcal{V}_{0}^{\mathbf{u}}$, we assume that

- the coefficients $\left(\Sigma_{1}, \Sigma_{2}, \Sigma_{3}\right)$ satisfy (1.4), the mobility satisfy (1.6), and the Cahn-Hilliard potential F satisfy (1.7),

- the discretization of non linear terms $\mathbf{d}^{F}$ satisfy (2.2) and the following property: there exists $K_{1}^{\mathbf{c}_{h}^{n}}>0$ (eventually depending on $\mathbf{c}_{h}^{n}$ ) such that

$$
\int_{\Omega}\left[F\left(\mathbf{a}_{h}^{n+1}\right)-F\left(\mathbf{c}_{h}^{n}\right)-\mathbf{d}^{F}\left(\mathbf{c}_{h}^{n}, \mathbf{a}_{h}^{n+1}\right) \cdot\left(\mathbf{a}_{h}^{n+1}-\mathbf{c}_{h}^{n}\right)\right] d x \leqslant K_{1}^{\mathbf{c}_{h}^{n}}, \forall \mathbf{a}_{h}^{n+1} \in \mathcal{V}_{\mathcal{S}}^{\mathbf{c}}
$$

Then, there exists at least one solution $\left(\mathbf{c}_{h}^{n+1}, \boldsymbol{\mu}_{h}^{n+1}, \mathbf{u}_{h}^{n+1}, p_{h}^{n+1}\right)$ to the problem 2.

The proof relies on the following lemma from the topological degree theory [10].

Lemma 3.3 (Topological degree). Let $W$ be a finite dimensional vector space, $\mathrm{G}$ be a continuous function from $W$ to $W$. Assume that there exists a continuous function $\mathrm{H}$ from $W \times[0 ; 1]$ to $W$ satisfying

(i) $\mathrm{H}(\cdot, 1)=\mathrm{G}$ and $\mathrm{H}(\cdot, 0)$ is affine,

(ii) $\exists R>0$ s.t. $\forall(w, \delta) \in W \times[0 ; 1]$, if $\mathrm{H}(w, \delta)=0$ then $|w|_{W}<R$,

(iii) the equation $\mathrm{H}(w, 0)=0$ has a solution $w \in W$,

Then there exists at least one solution $w \in W$ such that $\mathrm{G}(w)=0$ and $|w|_{W}<R$.

The idea is to link the non linear discrete problem to a more simple (linear) problem (using an homotopy, function $\mathrm{H}$ of lemma 3.3) for which we are able to prove existence of solutions (assumption (ii) of lemma 3.3). The topological degree theory allows to deduce the existence of solutions for the non linear problem from a priori estimates which are in our case deduced from the energy equality (3.1) proved in proposition 3.1. Such a methodology was used for the approximation of the triphasic Cahn-Hilliard model in [7]. We only give here the main steps of the proof.

$\alpha$ ) Problem 2 is reformulated to enter in the framework of lemma 3.3. Let $W$ be a finite dimensional vector space $\left(\mathcal{V}^{c}\right)^{2} \times\left(\mathcal{V}_{h}^{\mu}\right)^{2} \times \mathcal{V}_{h, 0}^{\mathbf{u}} \times \mathcal{V}^{p}$. We define a norm on $W$ : for all $w=\left(c_{1 h}, c_{2 h}, \mu_{1 h}, \mu_{2 h}, \mathbf{u}_{h}, p_{h}\right) \in W$,

$$
|w|_{W}^{2}=\left|c_{1 h}\right|_{\mathrm{H}^{1}(\Omega)}^{2}+\left|c_{2 h}\right|_{\mathrm{H}^{1}(\Omega)}^{2}+\left|\mu_{1 h}\right|_{\mathrm{H}^{1}(\Omega)}^{2}+\left|\mu_{2 h}\right|_{\mathrm{H}^{1}(\Omega)}^{2}+\left|\mathbf{u}_{h}\right|_{\left(\mathrm{H}^{1}(\Omega)\right)^{d}}^{2}+\left|p_{h}\right|_{\mathrm{L}^{2}(\Omega)}^{2},
$$

and we introduce the function $\mathrm{H}$ such that

$$
\begin{aligned}
\mathrm{H}: W \times[0 ; 1] & \rightarrow W \\
\left(w^{n+1}, \delta\right)=\left(c_{1 h}^{n+1}, c_{2 h}^{n+1}, \mu_{1 h}^{n+1}, \mu_{2 h}^{n+1} \mathbf{u}_{h}^{n+1}, p_{h}^{n+1}, \delta\right) & \mapsto\left(\mathcal{R}_{\delta}^{\mu_{1}}, \mathcal{R}_{\delta}^{c_{1}}, \mathcal{R}_{\delta}^{\mu_{2}}, \mathcal{R}_{\delta}^{c_{2}}, \mathcal{R}_{\delta}^{\mathbf{u}}, \mathcal{R}_{\delta}^{p}\right)
\end{aligned}
$$

where $\mathcal{R}_{\delta}^{c_{1}}$ and $\mathcal{R}_{\delta}^{c_{2}}$, (resp. $\mathcal{R}_{\delta}^{\mu_{1}}$ and $\mathcal{R}_{\delta}^{\mu_{2}}$, resp. $\mathcal{R}_{\delta}^{\mathbf{u}}$, resp. $\mathcal{R}_{\delta}^{p}$ ) are defined with their coordinates in the finite elements basis $\left(\nu_{I}^{c}\right)_{I \in \llbracket 1 ; \operatorname{dim}\left(\mathcal{V}_{h}^{c}\right) \rrbracket}\left(\operatorname{resp} .\left(\nu_{I}^{\mu}\right)_{I \in \llbracket 1 ; \operatorname{dim}\left(\mathcal{V}_{h}^{\mu}\right) \rrbracket}\right.$, resp. 
$\left(\boldsymbol{\nu}_{I}^{\mathbf{u}}\right)_{I \in \llbracket 1 ; \operatorname{dim}\left(\mathcal{V}_{h, 0}^{\mathrm{u}}\right) \rrbracket}$, resp. $\left.\left(\nu_{I}^{p}\right)_{I \in \llbracket 1 ; \operatorname{dim}\left(\mathcal{V}_{h}^{p}\right) \rrbracket}\right)$ of $\mathcal{V}_{h}^{c}\left(\right.$ resp. $\mathcal{V}_{h}^{\mu}$, resp. $\mathcal{V}_{h, 0}^{\mathbf{u}}$, resp. $\left.\mathcal{V}_{h}^{p}\right)$ :

$\forall I \in \llbracket 1 ; \operatorname{dim}\left(\mathcal{V}_{h}^{\mu}\right) \rrbracket$,

$$
\begin{aligned}
\left(\mathcal{R}_{\delta}^{\mu_{i}}\right)_{I} & =\int_{\Omega} \frac{c_{i h}^{n+1}-c_{i h}^{n}}{\Delta t} \nu_{I}^{\mu} d x+\int_{\Omega} \frac{M_{0 h \delta}^{n+\alpha}}{\Sigma_{i}} \nabla \mu_{i h}^{n+1} \cdot \nabla \nu_{I}^{\mu} d x \\
& -\delta \int_{\Omega}\left[c_{i h}^{n}-\alpha_{i h}\right]\left[\mathbf{u}_{h}^{n}-\frac{\Delta t}{\varrho_{h \delta}^{n}} \sum_{j=1}^{3}\left(c_{j h}^{n}-\alpha_{j h}\right) \nabla \mu_{j h}^{n+1}\right] \cdot \nabla \nu_{h}^{\mu} d x,
\end{aligned}
$$

$\forall I \in \llbracket 1 ; \operatorname{dim}\left(\mathcal{V}_{h}^{c}\right) \rrbracket$,

$$
\left(\mathcal{R}_{\delta}^{c_{i}}\right)_{I}=\int_{\Omega} \mu_{i h}^{n+1} \nu_{I}^{c} d x-\int_{\Omega} \delta D_{i}\left(\mathbf{c}_{h}^{n}, \mathbf{c}_{h}^{n+1}\right) \nu_{I}^{c} d x-\int_{\Omega} \frac{3}{4} \Sigma_{i} \varepsilon \nabla c_{i h}^{n+\beta} \cdot \nabla \nu_{I}^{c} d x,
$$

$\forall I \in \llbracket 1 ; \operatorname{dim}\left(\mathcal{V}_{h, 0}^{\mathbf{u}}\right) \rrbracket$,

$$
\begin{aligned}
\left(\mathcal{R}_{\delta}^{\mathbf{u}}\right)_{I} & =\int_{\Omega} \varrho_{h \delta}^{n} \frac{\mathbf{u}_{h}^{n+1}-\delta \mathbf{u}_{h}^{n}}{\Delta t} \boldsymbol{\nu}_{I}^{\mathbf{u}} d x+\frac{1}{2} \int_{\Omega} \frac{\varrho_{h \delta}^{n+1}-\varrho_{h \delta}^{n}}{\Delta t} \mathbf{u}_{h}^{n+1} \cdot \boldsymbol{\nu}_{I}^{\mathbf{u}} d x \\
& +\frac{\delta}{2} \int_{\Omega} \varrho_{h}^{n+1}\left(\mathbf{u}_{h}^{n} \cdot \nabla\right) \mathbf{u}_{h}^{n+1} \cdot \boldsymbol{\nu}_{I}^{\mathbf{u}} d x-\frac{\delta}{2} \int_{\Omega} \varrho_{h}^{n+1}\left(\mathbf{u}_{h}^{n} \cdot \nabla\right) \boldsymbol{\nu}_{I}^{\mathbf{u}} \cdot \mathbf{u}_{h}^{n+1} d x \\
& +\int_{\Omega} 2 \eta_{h \delta}^{n+1} D \mathbf{u}_{h}^{n+1}: D \boldsymbol{\nu}_{I}^{\mathbf{u}} d x-\int_{\Omega} p_{h}^{n+1} \operatorname{div}\left(\boldsymbol{\nu}_{I}^{\mathbf{u}}\right) d x \\
& -\int_{\Omega} \varrho_{h \delta}^{n+1} \mathbf{g} \cdot \boldsymbol{\nu}_{I}^{\mathbf{u}} d x+\delta \int_{\Omega} \sum_{j=1}^{3}\left(c_{j h}^{n}-\alpha_{j h}\right) \nabla \mu_{j h}^{n+1} \cdot \boldsymbol{\nu}_{I}^{\mathbf{u}} d x,
\end{aligned}
$$

$\forall I \in \llbracket 1 ; \operatorname{dim}\left(\mathcal{V}_{h}^{p}\right) \rrbracket, \quad\left(\mathcal{R}_{\delta}^{p}\right)_{I}=\int_{\Omega} \nu_{I}^{p} \operatorname{div}\left(\mathbf{u}_{h}^{n+1}\right) d x$,

with $M_{0 h \delta}^{n+\alpha}=M_{0}\left((1-\delta \alpha) \mathbf{c}_{h}^{n}+\delta \alpha \mathbf{c}_{h}^{n+1}\right), \varrho_{h \delta}^{\ell}=\varrho\left((1-\delta) \mathbf{c}_{h}^{\ell-1}+\delta \mathbf{c}_{h}^{\ell}\right)$ for $\ell=n$ or $\ell=n+1$ and $\eta_{h \delta}^{n+1}=\eta\left((1-\delta) \mathbf{c}_{h}^{n}+\delta \mathbf{c}_{h}^{n+1}\right)$. The function $\mathrm{G}$ is defined by $\mathrm{G}: w \in$ $W \rightarrow \mathrm{H}(w, 1) \in W$. The problem "Find $w^{n+1}$ such that $\mathrm{G}\left(w^{n+1}\right)=0$ " is equivalent (by definition of the function $\mathrm{H}$ ) to the problem 2. To prove the theorem, we are going to prove that the functions $\mathrm{H}$ and $\mathrm{G}$ satisfy the assumptions of lemma 3.3. The continuity of the function $\mathrm{H}$ is obtained using the continuity of the different non linear functions $\left(D_{i}^{F}, \varrho\right.$ and $\left.\eta\right)$ and the Lebesgue's theorem. The function $\mathrm{H}(\cdot, 0)$ is clearly affine by construction.

$\beta)$ Let $\left(w^{n+1}, \delta\right) \in W \times[0 ; 1]$ such that $\mathrm{H}\left(w^{n+1}, \delta\right)=0$. Note that $\mathrm{H}\left(w^{n+1}, \delta\right)=0$ is equivalent to say that $w^{n+1}=\left(c_{1 h}^{n+1}, c_{2 h}^{n+1}, \mu_{1 h}^{n+1}, \mu_{2 h}^{n+1}, \mathbf{u}_{h}^{n+1} p_{h}^{n+1}\right)$ is a solution of a problem closely related to the problem 2 . The same calculations as in the proof of proposition 3.1 allows to prove the following estimate:

$$
\begin{aligned}
& {\left[\mathcal{F}_{\Sigma, \varepsilon, \delta}^{\mathrm{triph}}\left(\mathbf{c}_{h}^{n+1}\right)+\frac{1}{2} \int_{\Omega} \varrho_{h \delta}^{n+1}\left|\mathbf{u}_{h}^{n+1}\right|^{2} d x\right]-\left[\mathcal{F}_{\Sigma, \varepsilon, \delta}^{\mathrm{triph}}\left(\mathbf{c}_{h}^{n}\right)+\frac{1}{2} \int_{\Omega} \varrho_{h \delta}^{n}\left|\delta \mathbf{u}_{h}^{n}\right|^{2} d x\right]} \\
& \quad+\Delta t \sum_{i=1}^{3} \int_{\Omega} \frac{M_{0 h}^{n+\alpha}}{\Sigma_{i}}\left|\nabla \mu_{i h}^{n+1}\right|^{2} d x+\Delta t \int_{\Omega} 2 \eta_{h \delta}^{n+1}\left|D \mathbf{u}_{h}^{n+1}\right|^{2} d x \\
& \quad+\frac{3}{8}(2 \beta-1) \varepsilon \int_{\Omega} \sum_{i=1}^{3} \Sigma_{i}\left|\nabla c_{i h}^{n+1}-\nabla c_{i h}^{n}\right|^{2} d x \\
& \quad+\frac{1}{2} \int_{\Omega} \varrho_{h \delta}^{n}\left[\left|\mathbf{u}_{h}^{n+1}-\mathbf{u}_{\delta}^{*}\right|_{\mathrm{L}^{2}(\Omega)}^{2}+\left|\mathbf{u}_{\delta}^{*}-\delta \mathbf{u}_{h}^{n}\right|_{\mathrm{L}^{2}(\Omega)}^{2}\right] d x \\
& \quad=\Delta t \int_{\Omega} \varrho_{h \delta}^{n+1} \mathbf{g} \cdot \mathbf{u}_{h}^{n+1} d x+\frac{12}{\varepsilon} \delta \int_{\Omega}\left[F\left(\mathbf{c}_{h}^{n+1}\right)-F\left(\mathbf{c}_{h}^{n}\right)-\mathbf{d}^{F}\left(\mathbf{c}_{h}^{n}, \mathbf{c}_{h}^{n+1}\right) \cdot\left(\mathbf{c}_{h}^{n+1}-\mathbf{c}_{h}^{n}\right)\right] d x
\end{aligned}
$$


where $\mathcal{F}_{\boldsymbol{\Sigma}, \varepsilon, \delta}^{\text {triph }}\left(\mathbf{c}_{h}^{\ell}\right)=\int_{\Omega} \delta \frac{12}{\varepsilon} F\left(\mathbf{c}_{h}^{\ell}\right)+\sum_{i=1}^{3} \frac{3}{8} \varepsilon \Sigma_{i}\left|\nabla c_{i h}^{\ell}\right|^{2} d x$. Using proposition 1.1, the fact that $F$ is non negative, the positive lower bounds $\varrho_{\min }$ and $\eta_{\min }$ for the density and viscosity, the fact that the mobility is bounded from below, the Korn lemma ( $c f$ [3, lemme VII.3.5]) and assumption (3.6), we can readily derive the following estimates

$$
\left|c_{i h}^{n+1}\right|_{\mathrm{H}^{1}(\Omega)}^{2} \leqslant K_{1}^{\mathbf{c}_{h}^{n}}, \quad\left|\mu_{i h}^{n+1}\right|_{\mathrm{H}^{1}(\Omega)}^{2} \leqslant K_{1}^{\mathbf{c}_{h}^{n}}, \quad\left|\mathbf{u}_{h}^{n+1}\right|_{\left(\mathrm{H}^{1}(\Omega)\right)^{d}} \leqslant K_{1}^{\mathbf{c}_{h}^{n}},
$$

where $K_{1}^{\mathbf{c}_{h}^{n}}$ is a constant independent of $\delta$ and $w^{n+1}$. The bound on pressure is obtained using the bound on the velocity (3.7) and the inf-sup condition (2.9) which ensures $\left(c f\left[8,21.5 .10\right.\right.$, p. 344]) that there exists $\mathbf{v}_{h} \in \mathcal{V}_{h, 0}^{\mathbf{u}}$ such that

$$
\forall \nu_{h}^{p} \in \mathcal{V}^{p}, \int_{\Omega} \nu_{h}^{p} \operatorname{div}\left(\mathbf{v}_{h}\right) d x=\int_{\Omega} \nu_{h}^{p} p_{h}^{n+1} d x \text { and }\left|\mathbf{v}_{h}\right|_{\left(\mathrm{H}^{1}(\Omega)\right)^{d}} \leqslant \frac{1}{\Theta}\left|p_{h}^{n+1}\right|_{\mathrm{L}^{2}(\Omega)} .
$$

Thus, taking $\boldsymbol{\nu}_{h}^{\mathbf{u}}=\mathbf{v}_{h}$ in the system associated to $\mathrm{H}\left(w^{n+1}, \delta\right)=0$ enables to bound the $\mathrm{L}^{2}$ norm of the pressure:

$$
\left|p_{h}^{n+1}\right|_{\mathrm{L}^{2}(\Omega)} \leqslant K_{2}^{\mathbf{c}_{h}^{n}}
$$

where $K_{2}^{\mathbf{c}_{h}^{n}}$ is a constant

Combining (3.7) and (3.9), we obtain a positive constant $K_{h}^{\mathbf{c}_{h}^{n}}$ independent of $\delta$ and $\mathbf{c}_{h}^{n+1}$ such that

$$
\left|w^{n+1}\right|_{W} \leqslant K^{\mathbf{c}_{h}^{n}}
$$

Hence, taking $R>K^{\mathbf{c}_{h}^{n}} \geqslant 0$ guarantees that for all $(w, \delta) \in W \times[0 ; 1], \mathrm{H}(w, \delta)=$ $0 \Longrightarrow|w|_{W}<R$.

$\gamma)$ It remains to prove the existence of a solution to the linear problem $\mathrm{H}\left(w^{n+1}, 0\right)=$ 0 . This problem can be written as three problems which are totally uncoupled and the existence of solutions for each of these problems is readily obtained (using inf-sup condition).

This concludes the proof of the existence of approximate solutions.

4. Numerical experiments. In this section, we provide 2D numerical simulations in order to illustrate the unconditional stability stated in Proposition 3.1.

The space discretization is performed on square local adaptive refined meshes using:

- $\mathbb{Q}_{1}$ Lagrange finite element for the order parameters $c_{1}, c_{2}, c_{3}$, the chemical potentials $\mu_{1}, \mu_{2}, \mu_{3}$ and for the pressure $p$,

- $\mathbb{Q}_{2}$ Lagrange finite element for each component of the velocity $\mathbf{u}$.

The adaptation procedures are based on conforming multilevel finite element approximation spaces which are built by refinement or unrefinement of the finite element basis functions instead of cells. All the details about this method and also various examples (in particular, simulations using the Cahn-Hilliard model considered in this article) are described in [5]. The refinement criterion used in those (un-)refinement procedures imposes the value of the smaller diameter $h_{\min }$ of a cell and ensures that refined areas are located in the neighborhood of the interfaces (i.e. where no order parameter is equal to one). We do not give more details on spatial discretization issues here since the main goal of this article is to investigate the properties of time discretization schemes. 
We compare the results obtained with the unconditionally stable scheme (denoted Uncond. in the sequel) proposed in this article (see Problem 2) and the scheme (denoted Stand. in the sequel) used in $[18,6]$ which is obtained by using an explicit velocity in the Cahn-Hilliard equation (see section 2.1.3).

The (nonlinear) Cahn-Hilliard system is solved using the Newton algorithm and the Navier-Stokes system is solved using the Augmented-Lagrangian method. All intermediates linear system are solved using direct solvers.

The practical implementation has been performed using the software objectoriented component library PELICANS [22], developed at the "Institut de Radioprotection et de Sûreté Nucléaire (IRSN)" and distributed under the CeCILL-C license agreement (an adaptation of LGPL to the French law).

4.1. Droplet oscillation. The first example is a two-phase flow simulation of the oscillations of a 2D droplet due to surface tension. This test case was already used in several articles, see for instance $[14,25,16,13]$. The initial configuration is a $2 \mathrm{D}$ droplet with a perturbed radius: $r=r_{0}(1+\alpha \cos (2 \theta))$ (in polar coordinates). More precisely, we choose the square $]-4 r_{0}, 4 r_{0}\left[^{2}\right.$ as computational domain and initialize the order parameters $c_{1}$ and $c_{2}$ with the following formula:

$$
\begin{aligned}
& c_{1}=0.5+0.5 \tanh \left(\frac{2}{\varepsilon}\left(r_{0}(1+\alpha \cos (2 \theta))-r\right)\right), \\
& c_{2}=1-c_{1},
\end{aligned}
$$

where the interface width $\varepsilon$ is given by $\varepsilon=\frac{r_{0}}{25}$. We use the values $r_{0}=0.1$ and $\alpha=0.05$ in all our simulations.

The perturbed droplet is initially at rest and the only external force is the surface tension $\sigma=1$ (i.e. there is no gravity $\mathbf{g}=0$ ). We assume that the two phases have the same densities $\varrho_{1}=\varrho_{2}=1$ and the same small viscosities $\eta_{1}=\eta_{2}=10^{-4}$. We take a small constant mobility $M_{0}=10^{-5}$ and perform simulations until the final time $T=0.2$. The space discretization is fixed: $h_{\min }=\frac{\varepsilon}{4}$.

Figure 4.1 shows the time evolution of the kinetic energy (on the left) and of the total energy (on the right) using the Uncond. scheme for different values of the time step $\Delta t$.

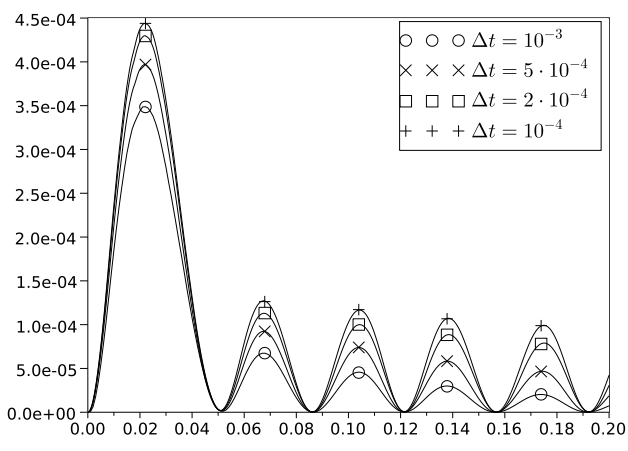

(a) Kinetic energy

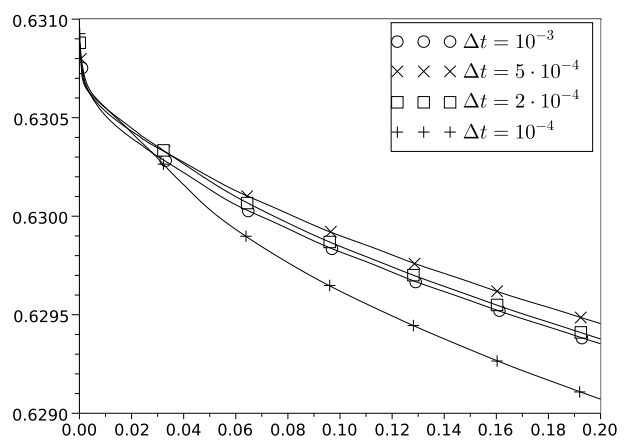

(b) Total energy

Fig. 4.1: Time evolution of kinetic and total energy using the Uncond. scheme for different time steps. 
The Uncond. scheme ensures the decrease of the total energy for all time steps. This is not the case when using Stand. scheme. Figure 4.2 shows a comparison of the energies evolution between Uncond. scheme and Stand. scheme. For $\Delta t=10^{-4}$, the results are very similar but for $\Delta t=2 \cdot 10^{-4}$ or greater, the Stand. scheme leads to a blow up of kinetic and total energies.

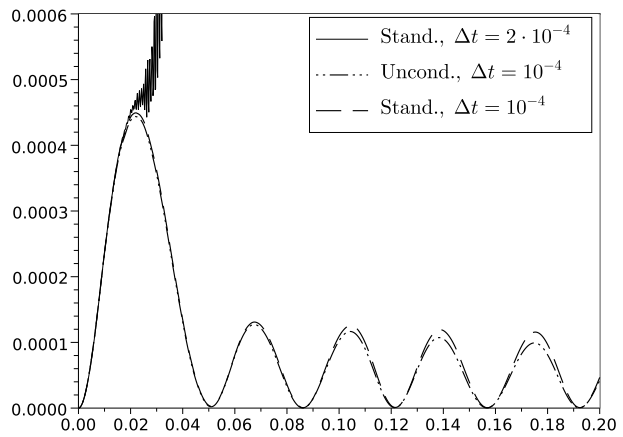

(a) Kinetic energy

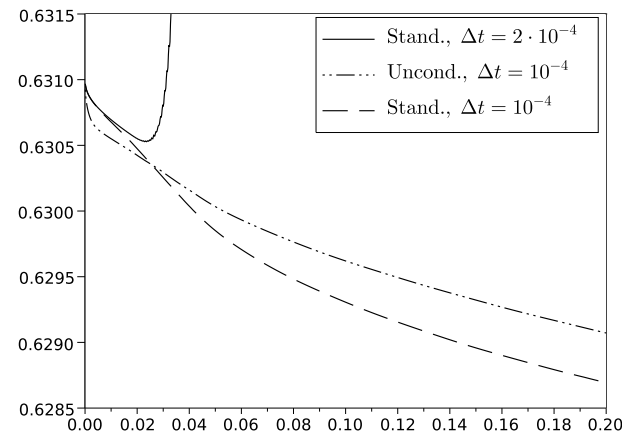

(b) Total energy

Fig. 4.2: Time evolution of kinetic and total energy using the Uncond. scheme and the Stand. scheme for different time steps.

Figure 4.3 shows the interface shape and the streamlines (of velocity) at $t=0.04$ that we obtain when using the Uncond. scheme (on the left) and the Stand. scheme (on the right). These pictures show 20 contour levels of the order parameter $c_{1}$ between 0.4 and 0.6 , and 50 contour levels of the streamline function. It appears small instabilities in the neighborhood of the interface when using the Stand. scheme.

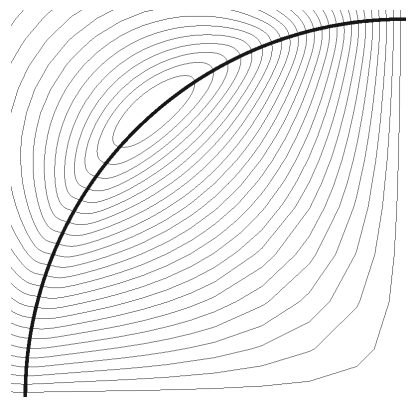

(a) Uncond. scheme

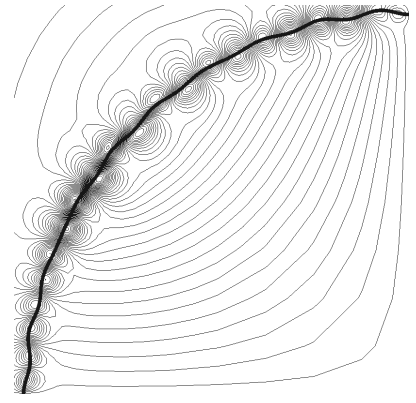

(b) Stand. scheme

Fig. 4.3: Contour level of the order parameter $c_{1}$ and of the velocity (streamlines) at $t=0.04$ using the two different schemes.

4.2. Two dimensional three-phase flow. The second example is a threephase flows simulation of a gas bubble rising in a liquid column under gravity. Physical properties of the three phases and initial configuration are given in Figure 4.4. The interface width is given by $\varepsilon=\frac{R}{15}$ and the mobility is chosen degenerate, i.e. the mobility vanishes in pure phases: $M_{0}(\mathbf{c})=10^{-5}\left(1-c_{1}\right)^{2}\left(1-c_{2}\right)^{2}\left(1-c_{3}\right)^{2}$. We chose 
$\Delta t=5 \cdot 10^{-5}$ and $h_{\min }=\frac{\varepsilon}{4}$ for this simulation.

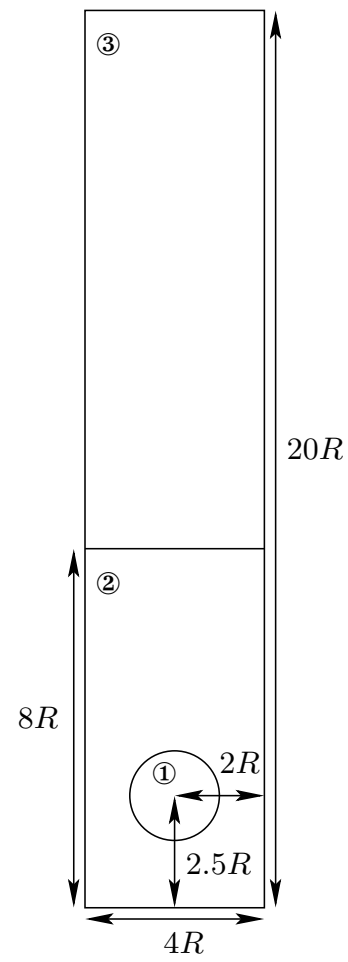

Fig. 4.4: Physical parameters and initial configuration of test case

The bubble rises in the heavy liquid (phase (2), penetrates the liquid-liquid interface and then rises in the light liquid (phase (3) entraining some quantity of the heavy liquid in the upper phase. This time evolution is shown in Figure 4.5 where simulations performed with the Uncond. scheme (on the left) and the Stand. scheme (on the right) are compared. These pictures show 50 contour levels of the order parameters $c_{1}$ (in red) and $c_{2}$ (in blue) between 0.4 and 0.6 (be careful the contour levels of $c_{1}$ and $c_{2}$ may coincide).

The results we obtain with the two schemes are close: the bubble rises with the same velocity in the two cases. However we observe two main differences: the first is the form of the bubble (see for instance picture at $t=0.6$ ) and the second is the time at which the column of entrained fluid break up (see pictures at time $t=0.75$ and $t=0.9$ ). These differences are certainly due to the fact that the stabilization modifies the value of the mobility in the Cahn-Hilliard equation and consequently the Uncond. scheme involves a more important diffusion at the interface than the standard scheme. Nevertheless, note that in the right picture at $t=0.9$, the thickness of the entrained liquid column is equal to the mesh size and so the column is near to break up.

5. Convergence of approximate solutions in the homogeneous case. In this section, we assume that $\varrho_{1}=\varrho_{2}=\varrho_{3}=\varrho_{0}>0$. This implies that the function $\varrho(\mathbf{c})$ is constant: $\varrho(\mathbf{c})=\varrho_{0}, \forall \mathbf{c} \in \mathcal{S}$.

The existence of solutions is given by theorem 3.2. For all $N \in \mathbb{N}$, we can introduce 


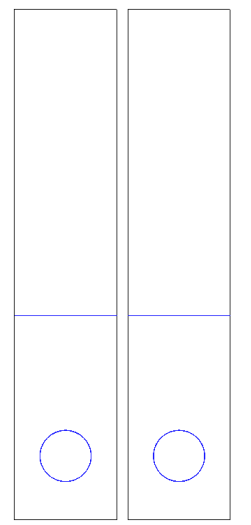

$t=0$

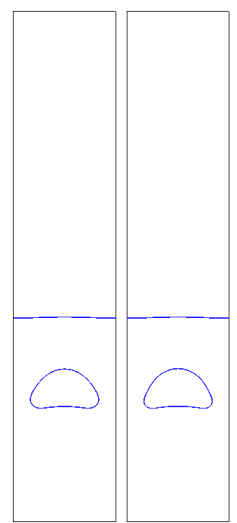

$t=0.15$

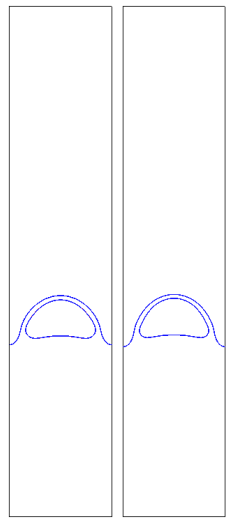

$t=0.3$

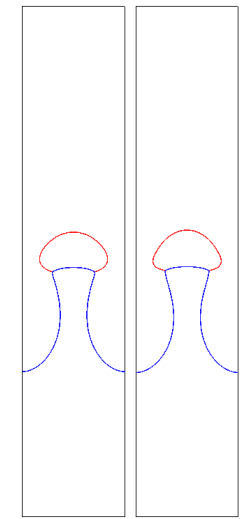

$t=0.45$

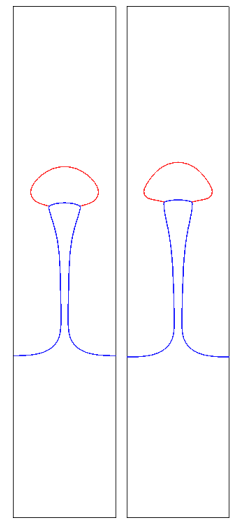

$t=0.6$

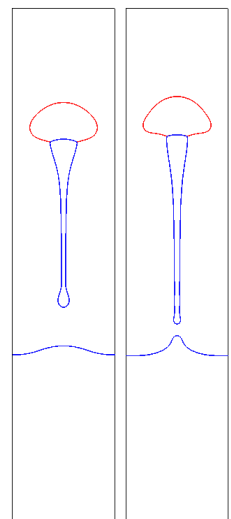

$t=0.75$

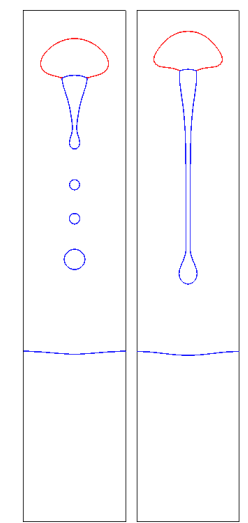

$t=0.9$

Fig. 4.5: Time evolution of the system with the Uncond. scheme on the left and with the Stand. scheme on the right.

the following piecewise constant and piecewise linear interpolations in time:

$$
\begin{gathered}
\left.\underline{c}_{i h}^{N}(t, \cdot)=c_{i h}^{n}(\cdot), \quad \bar{c}_{i h}^{N}(t, \cdot)=c_{i h}^{n+1}(\cdot), \quad \text { if } t \in\right] t_{n}, t_{n+1}[, \\
\left.c_{i h}^{N}(t, \cdot)=\frac{t_{n+1}-t}{\Delta t} c_{i h}^{n}(\cdot)+\frac{t-t_{n}}{\Delta t} c_{i h}^{n+1}(\cdot), \quad \text { if } t \in\right] t_{n}, t_{n+1}[. \\
\left.\mu_{i h}^{N}(t, \cdot)=\mu_{i h}^{n+1}(\cdot), \quad \text { if } t \in\right] t_{n}, t_{n+1}[. \\
\left.\underline{\mathbf{u}}_{h}^{N}(t, \cdot)=\mathbf{u}_{h}^{n}(\cdot), \quad \overline{\mathbf{u}}_{h}^{N}(t, \cdot)=\mathbf{u}_{h}^{n+1}(\cdot), \quad \text { if } t \in\right] t_{n}, t_{n+1}[, \\
\left.\mathbf{u}_{h}^{N}(t, \cdot)=\frac{t_{n+1}-t}{\Delta t} \mathbf{u}_{h}^{n}(\cdot)+\frac{t-t_{n}}{\Delta t} \mathbf{u}_{h}^{n+1}(\cdot), \quad \text { if } t \in\right] t_{n}, t_{n+1}[.
\end{gathered}
$$

The convergence result is the following: 
THEOREM 5.1 (Convergence theorem). We assume that assumptions of theorem 3.2 are satisfied, so that a solution $\left(\mathbf{c}_{h}^{N}, \boldsymbol{\mu}_{h}^{N}, \mathbf{u}_{h}^{N}, p_{h}^{N}\right)$ of problem 2 exists for all $N \in \mathbb{N}^{*}$ and for all $h>0$. We assume that $\left.\beta \in] \frac{1}{2}, 1\right]$, that the consistency property $(2.1)$ is satisfied and that there exist two constants $C>0$ and $\Delta t_{0}>0$ such that for all $\Delta t \leqslant \Delta t_{0}$ and for all $n \in \llbracket 0 ; N-1 \rrbracket$,

$$
\begin{aligned}
& {\left[\mathcal{F}_{\boldsymbol{\Sigma}, \varepsilon}^{\text {triph }}\left(\mathbf{c}_{h}^{n+1}\right)+\frac{1}{2} \varrho_{0} \int_{\Omega}\left|\mathbf{u}_{h}^{n+1}\right|^{2} d x\right]-\left[\mathcal{F}_{\boldsymbol{\Sigma}, \varepsilon}^{\text {triph }}\left(\mathbf{c}_{h}^{n}\right)+\frac{1}{2} \varrho_{0} \int_{\Omega}\left|\mathbf{u}_{h}^{n}\right|^{2} d x\right]} \\
& +C\left[\Delta t \sum_{i=1}^{3} \int_{\Omega} \frac{M_{0 h}^{n+\alpha}}{\Sigma_{i}}\left|\nabla \mu_{i h}^{n+1}\right|^{2} d x+\frac{3}{8}(2 \beta-1) \varepsilon \int_{\Omega} \sum_{i=1}^{3} \Sigma_{i}\left|\nabla c_{i h}^{n+1}-\nabla c_{i h}^{n}\right|^{2} d x\right] \\
& +\Delta t \int_{\Omega} 2 \eta_{h}^{n+1}\left|D \mathbf{u}_{h}^{n+1}\right|^{2} d x+\frac{1}{4} \varrho_{0} \int_{\Omega}\left|\mathbf{u}_{h}^{n+1}-\mathbf{u}_{h}^{n}\right|^{2} d x \leqslant \Delta t \varrho_{0} \int_{\Omega} \mathbf{g} \cdot \mathbf{u}_{h}^{n+1} d x .
\end{aligned}
$$

Consider the problem (1.8), the initial conditions (1.11) and the boundary conditions (1.9)-(1.10). Then, for all $t_{f}>0$, there exists a weak solution $(\mathbf{c}, \boldsymbol{\mu}, \mathbf{u}, p)$ defined on $\left[0, t_{f}[\right.$ such that

$$
\begin{gathered}
\mathbf{c} \in \mathrm{L}^{\infty}\left(0, t_{f} ;\left(\mathrm{H}^{1}(\Omega)\right)^{3}\right) \cap \mathcal{C}^{0}\left(\left[0, t_{f}\left[;\left(\mathrm{L}^{q}(\Omega)\right)^{3}\right), \text { for all } q<6\right.\right. \\
\boldsymbol{\mu} \in \mathrm{L}^{2}\left(0, t_{f} ;\left(\mathrm{H}^{1}(\Omega)\right)^{3}\right), \\
\mathbf{u} \in \mathrm{L}^{\infty}\left(0, t_{f} ;\left(\mathrm{L}^{2}(\Omega)\right)^{d}\right) \cap \mathrm{L}^{2}\left(0, t_{f} ;\left(\mathrm{H}^{1}(\Omega)\right)^{d}\right), \\
\mathbf{c}(t, x) \in \mathcal{S}, \text { for almost every }(t, x) \in\left[0, t_{f}[\times \Omega .\right.
\end{gathered}
$$

Moreover, for all sequences $\left(h_{K}\right)_{K \in \mathbb{N}^{*}}$ and $\left(N_{K}\right)_{K \in \mathbb{N}^{*}}$ satisfying the following properties:

- $h_{K} \underset{K \rightarrow+\infty}{\longrightarrow} 0$ and $N_{K} \underset{K \rightarrow+\infty}{\longrightarrow}+\infty$,

- there exists a constant $A$ (indep. of $K$ ) s.t.: (recall that $\mathrm{C}_{\mathrm{inv}}$ is given by (2.8))

$$
\forall K \in \mathbb{N}^{*}, \mathrm{C}_{\mathrm{inv}}\left(h_{K}\right) \leqslant A N_{K},
$$

the sequences $\left(\mathbf{c}_{h_{K}}^{N_{K}}\right)_{K \in \mathbb{N}^{*}},\left(\boldsymbol{\mu}_{h_{K}}^{N_{K}}\right)_{K \in \mathbb{N}^{*}}$ and $\left(\mathbf{u}_{h_{K}}^{N_{K}}\right)_{K \in \mathbb{N}^{*}}$ satisfy, up to a subsequence, the following convergences when $K \longrightarrow+\infty$ :

$$
\begin{aligned}
\mathbf{c}_{h_{K}}^{N_{K}} & \rightarrow \mathbf{c} \text { in } \mathcal{C}^{0}\left(0, t_{f},\left(\mathrm{~L}^{q}\right)^{3}\right) \text { strong, for all } q<6, \\
\mathbf{u}_{h_{K}}^{N_{K}} & \rightarrow \mathbf{u} \text { in } \mathrm{L}^{2}\left(0, t_{f},\left(\mathrm{~L}^{2}\right)^{d}\right) \text { strong, } \\
\boldsymbol{\mu}_{h_{K}}^{N_{K}} & \rightarrow \boldsymbol{\mu} \text { in } \mathrm{L}^{2}\left(0, t_{f},\left(\mathrm{H}^{1}\right)^{3}\right) \text { weak. }
\end{aligned}
$$

REMARK 4. The assumption (5.1) is obtained in practice by applying the proposition 3.1 and by bounding the term:

$$
\int_{\Omega}\left[F\left(\mathbf{c}_{h}^{n+1}\right)-F\left(\mathbf{c}_{h}^{n}\right)-\mathbf{d}^{F}\left(\mathbf{c}_{h}^{n}, \mathbf{c}_{h}^{n+1}\right) \cdot\left(\mathbf{c}_{h}^{n+1}-\mathbf{c}_{h}^{n}\right)\right] d x,
$$

in the right hand side of (3.1). The way to obtain this bound is depends on the scheme $D_{i}^{F}\left(\mathbf{c}^{n}, \mathbf{c}^{n+1}\right)$ chosen for the non linear terms of the Cahn-Hilliard system. This was largely discussed in reference [7].

REMARK 5. In the statement of theorem 5.1, the inequality (5.2) is not a stability condition. When using a quasi-uniform mesh family and the associated Lagrange finite elements in 2D, this condition is not restrictive since we can choose $\mathrm{C}_{\mathrm{inv}}(h)=$ $\mathrm{C}(1+|\ln (h)|)$. In 3D, it means that to obtain convergence towards weak solution of continuous problem, it is necessary that the time step goes to zero faster than the mesh size. 
The proof of theorem 5.1 is inspired from the references [12] and [18] which deal with the homogeneous diphasic Cahn-Hilliard/Navier-Stokes system. Excluding the fact that we are interesting in a triphasic model, the major difference with these works is the taking into account of the transport term in the Cahn-Hilliard equation. We have to prove that the additional term do not disturb the consistency.

Basically, the proof of theorem 5.1 is split in three steps:

- first, the energy equality (5.1) allows to prove that the sequences $\left(\mathbf{c}_{h_{K}}^{N_{K}}\right)_{K \in \mathbb{N}^{*}}$, $\left(\boldsymbol{\mu}_{h_{K}}^{N_{K}}\right)_{K \in \mathbb{N}^{*}}$ and $\left(\mathbf{u}_{h_{K}}^{N_{K}}\right)_{K \in \mathbb{N}^{*}}$ are bounded in some suitable norms.

- it is then possible to apply compactness theorems to extract some convergent subsequences.

- the third step consists in proving that the obtained limit is a weak solution of problem (1.8).

We give the details for each of these three steps below. In the sequel (section 5.1, 5.2 and 5.3), we assume that assumptions of theorem 5.1 are satisfied and in particular the notation $\mathbf{c}_{h}^{n}, \boldsymbol{\mu}_{h}^{n}, \mathbf{u}_{h}^{n}, p_{h}^{n} \ldots$ denote solutions of the discrete problem 2 , and $\left(\mathbf{c}_{h_{K}}^{N_{K}}\right)_{K \in \mathbb{N}^{*}}$, $\left(\boldsymbol{\mu}_{h_{K}}^{N_{K}}\right)_{K \in \mathbb{N}^{*}},\left(\mathbf{u}_{h_{K}}^{N_{K}}\right)_{K \in \mathbb{N}^{*}} \ldots$ the associated sequences.

5.1. Bounds on discrete solution. In this section, we assume that $K$ is fixed and to simplify notation we omit the index $K$ in the notation $h_{K}$ and $N_{K}$. The first estimates stated in proposition 5.2 are directly derived from the energy estimate (5.1).

Proposition 5.2. We have the following bounds:

$$
\begin{array}{r}
\sup _{n \leqslant N}\left(\left|\mathbf{c}_{h}^{n}\right|_{\left(\mathrm{H}^{1}(\Omega)\right)^{3}}\right)+\sup _{n \leqslant N}\left(\left|\mathbf{u}_{h}^{n}\right|_{\left(\mathrm{L}^{2}(\Omega)\right)^{d}}\right) \\
\left(\sum_{n=0}^{N-1} \Delta t \sum_{i=1}^{3}\left|\mu_{i h}^{n+1}\right|_{\mathrm{H}^{1}(\Omega)}^{2}\right)+\left(\sum_{n=0}^{N-1} \Delta t\left|\mathbf{u}_{h}^{n+1}\right|_{\left(\mathrm{H}^{1}(\Omega)\right)^{d}}^{2}\right) \leqslant K_{2}, \\
\Delta t\left(\sum_{n=0}^{N-1} \Delta t \sum_{i=1}^{3}\left|\frac{c_{i h}^{n+1}-c_{i h}^{n}}{\Delta t}\right|_{\mathrm{H}^{1}(\Omega)}^{2}\right)+\left(\sum_{n=0}^{N-1}\left|\mathbf{u}_{h}^{n+1}-\mathbf{u}_{h}^{n}\right|_{\left(\mathrm{L}^{2}(\Omega)\right)^{d}}^{2}\right)
\end{array}
$$

where $K_{1}, K_{2}$ and $K_{3}$ are three constants independent of $\Delta t$ and $h$.

Proof. This proof is very similar to the one of proposition 4.2 in [7]. Nevertheless, note that it use additional ingredients (Korn lemma (cf [3, lemma VII.3.5]), the lower bound for the viscosity $\eta(c)$ and the fact that the density is constant) to deal with the terms which involve the velocity.

To pass to the limit in non linear equations ( $c f$ section 5.3), we need strong convergence of the subsequences. For this reason, it is useful to obtain more accurate estimates.

Proposition 5.3. There exist two constants $K_{4}$ and $K_{5}$ independent of $h$ and $\Delta t$ such that:

$$
\begin{aligned}
& \left(\sum_{n=0}^{N-1} \Delta t \sum_{i=1}^{3}\left|\frac{c_{i h}^{n+1}-c_{i h}^{n}}{\Delta t}\right|_{\left(\mathrm{H}^{1}(\Omega)\right)^{\prime}}^{2}\right)+\left(\frac{1}{\sqrt{\Delta t}} \sum_{n=0}^{N-1} \sum_{i=1}^{3}\left|c_{i h}^{n+1}-c_{i h}^{n}\right|_{\left(\mathrm{L}^{2}(\Omega)\right)}^{2}\right) \leqslant K_{4}, \\
& \sum_{n=0}^{N-i-1} \Delta t\left|\mathbf{u}^{n+i}-\mathbf{u}^{n}\right|_{\left(\mathrm{L}^{2}(\Omega)\right)^{d}}^{2} \leqslant K_{5}\left(t^{i}\right)^{\frac{1}{4}}, \quad \forall i \in \llbracket 0 ; N-1 \rrbracket .
\end{aligned}
$$

Proof.

(i) The estimate (5.6) is obtained from the first equation of the Cahn-Hilliard system. 
( $\alpha$ ) Consider $\nu_{h}^{\mu} \in \mathcal{V}_{h}^{\mu}$. The first equation of (2.3) reads:

$$
\begin{aligned}
\int_{\Omega} \frac{c_{i h}^{n+1}-c_{i h}^{n}}{\Delta t} & \nu_{h}^{\mu} d x=-\int_{\Omega} \frac{M_{0 h}^{n+\alpha}}{\Sigma_{i}} \nabla \mu_{i h}^{n+1} \cdot \nabla \nu_{h}^{\mu} d x \\
& +\int_{\Omega}\left[c_{i h}^{n}-\alpha_{i}\right]\left[\mathbf{u}_{h}^{n}-\frac{\Delta t}{\varrho_{0}} \sum_{j=1}^{3}\left(c_{j h}^{n}-\alpha_{j}\right) \nabla \mu_{j h}^{n+1}\right] \cdot \nabla \nu_{h}^{\mu} d x .
\end{aligned}
$$

Thus, the inverse inequality (2.8) yields:

$$
\begin{aligned}
& \left|\int_{\Omega} \frac{c_{i h}^{n+1}-c_{i h}^{n}}{\Delta t} \nu_{h}^{\mu} d x\right| \leqslant\left[\frac{M_{2}}{\Sigma_{m}}\left|\mu_{i h}^{n+1}\right|_{\mathrm{H}^{1}}+\left(\left|\alpha_{i}\right|+\left|c_{i h}^{n}\right|_{\mathrm{H}^{1}}\right)\left|\mathbf{u}_{h}^{n}\right|_{\mathrm{H}^{1}}\right]\left|\nu_{h}^{\mu}\right|_{\mathrm{H}^{1}} \\
& \quad+\frac{\Delta t}{\varrho_{0}}\left(\left|\alpha_{i}\right|+\mathrm{C}_{\mathrm{inv}}(h)^{\frac{1}{2}}\left|c_{i h}^{n}\right|_{\mathrm{H}^{1}}\right) \sum_{j=1}^{3}\left(1+\mathrm{C}_{\mathrm{inv}}(h)^{\frac{1}{2}}\left|c_{j h}^{n}\right|_{\mathrm{H}^{1}}\right)\left|\mu_{j h}^{n+1}\right|_{\mathrm{H}^{1}}\left|\nu_{h^{\mu}}^{\mu}\right|_{\mathrm{H}^{1}} .
\end{aligned}
$$

Finally, thanks to (5.2) and (5.3), we obtained that there exists a constant $K$ (independent of $h$ and $\Delta t$ ) such that:

$$
\left|\int_{\Omega} \frac{c_{i h}^{n+1}-c_{i h}^{n}}{\Delta t} \nu_{h}^{\mu} d x\right| \leqslant K\left[\left|\mathbf{u}_{h}^{n}\right|_{\mathrm{H}^{1}(\Omega)}+\sum_{i=1}^{3}\left|\mu_{i h}^{n+1}\right|_{\mathrm{H}^{1}(\Omega)}\right]\left|\nu_{h}^{\mu}\right|_{\mathrm{H}^{1}(\Omega)} .
$$

We are now going to use this intermediate inequality to prove (5.6).

( $\beta)$ Let $\nu \in \mathrm{H}^{1}(\Omega)$. Let $\nu_{h}^{\mu}$ be the $\mathrm{L}^{2}$ projection of $\nu$ on $\mathcal{V}_{h}^{\mu}$. Owing to (2.7), we have:

$$
\left|\nu_{h}^{\mu}\right|_{\mathrm{H}^{1}(\Omega)} \leqslant C|\nu|_{\mathrm{H}^{1}(\Omega)} .
$$

Thus, using (5.8), we obtain

$$
\left|\int_{\Omega} \frac{c_{i h}^{n+1}-c_{i h}^{n}}{\Delta t} \nu d x\right|=\left|\int_{\Omega} \frac{c_{i h}^{n+1}-c_{i h}^{n}}{\Delta t} \nu_{h}^{\mu} d x\right| \leqslant K C\left[\left|\mathbf{u}_{h}^{n}\right|_{\mathrm{H}^{1}}+\sum_{i=1}^{3}\left|\mu_{i h}^{n+1}\right|_{\mathrm{H}^{1}}\right]|\nu|_{\mathrm{H}^{1}} .
$$

Since this inequality is true for all $\nu \in \mathrm{H}^{1}(\Omega)$, we have

$$
\left|\frac{c_{i h}^{n+1}-c_{i h}^{n}}{\Delta t}\right|_{\left(\mathrm{H}^{1}(\Omega)\right)^{\prime}} \leqslant K C\left[\left|\mathbf{u}_{h}^{n}\right|_{\mathrm{H}^{1}(\Omega)}+\sum_{i=1}^{3}\left|\mu_{i h}^{n+1}\right|_{\mathrm{H}^{1}(\Omega)}\right] .
$$

Consequently, using (5.4) yields:

$$
\sum_{n=0}^{N-1} \Delta t \sum_{i=1}^{3}\left|\frac{c_{i h}^{n+1}-c_{i h}^{n}}{\Delta t}\right|_{\left(\mathrm{H}^{1}(\Omega)\right)^{\prime}}^{2} \leqslant 18 K^{2} C^{2} K_{2} .
$$

$(\gamma)$ We now take $\nu_{h}^{\mu}=\Delta t\left(c_{i h}^{n+1}-c_{i h}^{n}\right)$ in (5.8). This yields:

$$
\sum_{i=1}^{3}\left|\int_{\Omega}\right| c_{i h}^{n+1}-\left.c_{i h}^{n}\right|^{2} d x\left|\leqslant K \Delta t\left[\left|\mathbf{u}_{h}^{n}\right|_{\mathrm{H}^{1}}+\sum_{i=1}^{3}\left|\mu_{i h}^{n+1}\right|_{\mathrm{H}^{1}}\right] \sum_{i=1}^{3}\right| c_{i h}^{n+1}-\left.c_{i h}^{n}\right|_{\mathrm{H}^{1}} .
$$

and so, using (5.4) and (5.5), we have:

$$
\sum_{n=0}^{N-1} \sum_{i=1}^{3}\left|c_{i h}^{n+1}-c_{i h}^{n}\right|_{\mathrm{L}^{2}(\Omega)}^{2} \leqslant 2 \sqrt{K_{2}} \sqrt{K_{3}} \sqrt{\Delta t} .
$$


The inequality (5.6) is readily deduced from equations (5.9) and (5.10) by defining the constant $K_{4}=\max \left(18 K^{2} C^{2} K_{2}, 2 \sqrt{3 K_{2}} \sqrt{K_{3}}\right)$.

(ii) To obtain estimate (5.7), we begin with bounding the term: $\left|\mathbf{u}_{h}^{n+i}-\mathbf{u}_{h}^{n}\right|_{\left(\mathrm{L}^{2}\right)^{d}}^{2}$ for $n \in \llbracket 0 ; N-i-1 \rrbracket$. We choose $\boldsymbol{\nu}_{h}^{\mathbf{u}} \in \mathcal{V}_{h, 0}^{\mathbf{u}}$ such that

$$
\int_{\Omega} \nu_{h}^{p} \operatorname{div} \boldsymbol{\nu}_{h}^{\mathbf{u}} d x=0, \quad \forall \nu_{h}^{p} \in \mathcal{V}^{p}
$$

as test function in (2.11) and we sum up the equations to obtain:

$$
\begin{aligned}
\int_{\Omega} \varrho_{0}\left(\mathbf{u}_{h}^{n+i}-\mathbf{u}_{h}^{n}\right) \cdot \boldsymbol{\nu}_{h}^{\mathbf{u}} d x+\underbrace{\frac{1}{2} \sum_{k=n}^{n+i-1} \Delta t \int_{\Omega} \varrho_{0}\left(\mathbf{u}_{h}^{k} \cdot \nabla\right) \mathbf{u}_{h}^{k+1} \cdot \boldsymbol{\nu}_{h}^{\mathbf{u}} d x}_{T_{1}} \\
-\underbrace{\frac{1}{2} \sum_{k=n}^{n+i-1} \Delta t \int_{\Omega} \varrho_{0}\left(\mathbf{u}_{h}^{k} \cdot \nabla\right) \boldsymbol{\nu}_{h}^{\mathbf{u}} \cdot \mathbf{u}_{h}^{k+1} d x}_{T_{2}}+\underbrace{\sum_{k=n}^{n+i-1} \Delta t \int_{\Omega} 2 \eta_{h}^{k+1} D \mathbf{u}_{h}^{k+1}: D \boldsymbol{\nu}_{h}^{\mathbf{u}} d x}_{T_{4}} \\
=\underbrace{\sum_{k=n}^{n+i-1} \Delta t \int_{\Omega} \varrho_{0} \mathbf{g} \cdot \boldsymbol{\nu}_{h}^{\mathbf{u}} d x}_{T_{3}}-\underbrace{\sum_{k=n}^{n+i-1} \Delta t \int_{\Omega} \sum_{j=1}^{3}\left(c_{j h}^{k}-\alpha_{j}\right) \nabla \mu_{j h}^{k+1} \cdot \boldsymbol{\nu}_{h}^{\mathbf{u}} d x}_{T_{5}} .
\end{aligned}
$$

We then separately estimate each term of this inequality. For term $T_{1}$, by using the Hölder inequality and an interpolation inequality, we obtain:

$$
T_{1} \leqslant \frac{1}{2} \varrho_{0}\left|\boldsymbol{\nu}_{h}^{\mathbf{u}}\right|_{\left(\mathrm{H}^{1}\right)^{d}} \Delta t \sum_{k=n}^{n+i-1}\left|\mathbf{u}^{k}\right|_{\left(\mathrm{L}^{2}\right)^{d}}^{\frac{1}{2}}\left|\mathbf{u}^{k}\right|_{\left(\mathrm{L}^{6}\right)^{d}}^{\frac{1}{2}}\left|\mathbf{u}^{k+1}\right|_{\left(\mathrm{H}^{1}\right)^{d}} .
$$

Using the bound (5.3) and the Young inequality yields:

$$
T_{1} \leqslant \frac{1}{2} \varrho_{0} K_{1}^{\frac{1}{2}}\left|\nu_{h}^{\mathbf{u}}\right|_{\left(\mathrm{H}^{1}\right)^{d}} \Delta t \sum_{k=n}^{n+i-1} \frac{2}{3}\left[\left|\mathbf{u}^{k}\right|_{\left(\mathrm{H}^{1}\right)^{d}}^{\frac{3}{2}}+\left|\mathbf{u}^{k+1}\right|_{\left(\mathrm{H}^{1}\right)^{d}}^{\frac{3}{2}}\right]
$$

We conclude by using the Hölder inequality and the bound (5.4):

$$
T_{1} \leqslant \frac{2}{3} \varrho_{0} K_{1}^{\frac{1}{2}} K_{2}^{\frac{3}{4}}\left|\boldsymbol{\nu}_{h}^{\mathbf{u}}\right|_{\left(\mathrm{H}^{1}\right)^{d}}\left(t_{f}\right)^{\frac{3}{4}}\left(t^{i}\right)^{\frac{1}{4}}
$$

The term $T_{2}$ is bounded in the same way:

$$
\begin{aligned}
& T_{2} \leqslant \frac{1}{2} \varrho_{0} \Delta t \sum_{k=n}^{n+i-1}\left|\mathbf{u}^{k}\right|_{\left(\mathrm{L}^{3}\right)^{d}}\left|\boldsymbol{\nu}_{h}^{\mathbf{u}}\right|_{\left(\mathrm{H}^{1}\right)^{d}}\left|\mathbf{u}^{k+1}\right|_{\left(\mathrm{L}^{6}\right)^{d}} \\
& \leqslant \frac{1}{2} \varrho_{0}\left|\boldsymbol{\nu}_{h}^{\mathbf{u}}\right|_{\left(\mathrm{H}^{1}\right)^{d}} \Delta t \sum_{k=n}^{n+i-1}\left|\mathbf{u}^{k}\right|_{\left(\mathrm{L}^{2}\right)^{d}}^{\frac{1}{2}}\left|\mathbf{u}^{k}\right|_{\left(\mathrm{L}^{6}\right)^{d}}^{\frac{1}{2}}\left|\mathbf{u}^{k+1}\right|_{\left(\mathrm{H}^{1}\right)^{d}} \\
& \leqslant \frac{2}{3} \varrho_{0} K_{1}^{\frac{1}{2}} K_{2}^{\frac{3}{4}}\left|\boldsymbol{\nu}_{h}^{\mathbf{u}}\right|_{\left(\mathrm{H}^{1}\right)^{d}}\left(t_{f}\right)^{\frac{3}{4}}\left(t^{i}\right)^{\frac{1}{4}} \\
& 24
\end{aligned}
$$


For the viscous term $T_{3}$, we derive the following estimate:

$$
\begin{aligned}
T_{3} & \leqslant 2 \eta_{\max } \Delta t \sum_{k=n}^{n+i-1}\left|\mathbf{u}_{h}^{k+1}\right|_{\left(\mathrm{H}^{1}\right)^{d}}\left|\boldsymbol{\nu}_{h}^{\mathbf{u}}\right|_{\left(\mathrm{H}^{1}\right)^{d}} \\
& \leqslant 2 \eta_{\max }\left|\boldsymbol{\nu}_{h}^{\mathbf{u}}\right|_{\left(\mathrm{H}^{1}\right)^{d}} \Delta t i^{\frac{1}{2}}\left(\sum_{k=n}^{n+i-1}\left|\mathbf{u}_{h}^{k+1}\right|_{\left(\mathrm{H}^{1}\right)^{d}}^{2}\right)^{\frac{1}{2}} \\
& \leqslant 2 \eta_{\max } K_{2}^{\frac{1}{2}}\left|\boldsymbol{\nu}_{h}^{\mathbf{u}}\right|_{\left(\mathrm{H}^{1}\right)^{d}}\left(t_{f}\right)^{\frac{1}{2}}\left(t^{i}\right)^{\frac{1}{2}}
\end{aligned}
$$

It remains the terms $T_{4}$ and $T_{5}$ :

$$
T_{4} \leqslant \varrho_{0}|\mathbf{g}|_{2}|\Omega|^{\frac{1}{2}}\left|\nu_{h}^{\mathbf{u}}\right|_{\mathrm{L}^{2}} t^{i}
$$

and

$$
\begin{aligned}
T_{5} & \leqslant \sum_{k=n}^{n+i-1} \Delta t \sum_{j=1}^{3}\left|c_{j h}^{k}-\alpha_{j}\right|_{\mathrm{L}^{4}}\left|\mu_{j h}^{k+1}\right|_{\mathrm{H}^{1}}\left|\boldsymbol{\nu}_{h}^{\mathbf{u}}\right|_{\left(\mathrm{L}^{4}\right)^{d}} \\
& \leqslant|\Omega|\left[K_{1}+\max _{i=1,2,3}\left|\alpha_{i}\right|\right] K_{2}^{\frac{1}{2}}\left|\boldsymbol{\nu}_{h}^{\mathbf{u}}\right|_{\left(\mathrm{H}^{1}\right)^{d}}\left(t^{i}\right)^{\frac{1}{2}} .
\end{aligned}
$$

Finally, we obtain the following result: there exists a positive constant $K$ such that, for all $\boldsymbol{\nu}_{h}^{\mathbf{u}} \in \mathcal{V}_{h, 0}^{\mathbf{u}}$ satisfying (5.11), we have

$$
\left|\int_{\Omega}\left(\mathbf{u}_{h}^{n+i}-\mathbf{u}_{h}^{n}\right) \cdot \boldsymbol{\nu}_{h}^{\mathbf{u}} d x\right| \leqslant K\left|\boldsymbol{\nu}_{h}^{\mathbf{u}}\right|_{\left(\mathrm{H}^{1}\right)^{d}}\left(t^{i}\right)^{\frac{1}{4}} .
$$

In particular, for $\boldsymbol{\nu}_{h}^{\mathbf{u}}=\mathbf{u}_{h}^{n+i}-\mathbf{u}_{h}^{n}$ (which satisfies (5.11) owing to (2.11)), we find

$$
\left|\mathbf{u}_{h}^{n+i}-\mathbf{u}_{h}^{n}\right|_{\left(\mathrm{L}^{2}\right)^{d}}^{2} \leqslant K\left|\mathbf{u}_{h}^{n+i}-\mathbf{u}_{h}^{n}\right|_{\left(\mathrm{H}^{1}\right)^{d}}\left(t^{i}\right)^{\frac{1}{4}} .
$$

Thus, we obtain

$$
\sum_{n=0}^{N-i-1} \Delta t\left|\mathbf{u}_{h}^{n+i}-\mathbf{u}_{h}^{n}\right|_{\left(\mathrm{L}^{2}(\Omega)\right)^{d}}^{2} \leqslant 2 K\left(t_{f}\right)^{\frac{1}{2}}\left(t^{i}\right)^{\frac{1}{4}} \sum_{n=0}^{N-1} \Delta t\left|\mathbf{u}_{h}^{n}\right|_{\left(\mathrm{H}^{1}(\Omega)\right)^{d}}^{2} .
$$

This leads to the conclusion with $K_{5}=2 K K_{2}\left(t_{f}\right)^{\frac{1}{2}}$.

5.2. Compactness argument, convergence of subsequences. The estimates proved in section 5.1 (proposition 5.2 and 5.3), allow to obtain (up to subsequences) the convergence of the sequences: $\mathbf{c}_{h_{K}}^{N_{K}}, \overline{\mathbf{c}}_{h_{K}}^{N_{K}}, \underline{\mathbf{c}}_{h_{K}}^{N_{K}}, \boldsymbol{\mu}_{h_{K}}^{N_{K}}, \mathbf{u}_{h_{K}}^{N_{K}}, \overline{\mathbf{u}}_{h_{K}}^{N_{K}}$ and $\underline{\mathbf{u}}_{h_{K}}^{N_{K}}$. The following propositions give the spaces in which these convergences hold.

Proposition 5.4. Up to subsequences, we have the following convergences when $K \rightarrow+\infty$ :

$$
\begin{array}{cc}
\mathbf{c}_{h_{K}}^{N_{K}} \rightarrow \mathbf{c} & \text { in } \mathrm{L}^{\infty}\left(0, t_{f},\left(\mathrm{H}^{1}(\Omega)\right)^{3}\right) \text { weak-*, } \\
\boldsymbol{\mu}_{h_{K}}^{N_{K}} \rightarrow \boldsymbol{\mu} & \text { in } \mathrm{L}^{2}\left(0, t_{f},\left(\mathrm{H}^{1}(\Omega)\right)^{3}\right) \text { weak, } \\
\frac{\partial \mathbf{c}_{h_{K}}^{N_{K}}}{\partial t} \rightarrow \frac{\partial \mathbf{c}}{\partial t} & \text { in } \mathrm{L}^{2}\left(0, t_{f},\left(\mathrm{H}^{1}(\Omega)\right)^{\prime}\right) \text { weak }, \\
\mathbf{u}_{h_{K}}^{N_{K}} \rightarrow \mathbf{u} & \text { in } \mathrm{L}^{2}\left(0, t_{f},\left(\mathrm{H}^{1}(\Omega)\right)^{d}\right) \text { weak. }
\end{array}
$$


Proof. The convergences (5.12), (5.13), (5.14) and (5.15) are direct consequences of proposition 5.2. Indeed, it is easy to verify that the estimates stated in this proposition prove that the sequences $\mathbf{c}_{h_{K}}^{N_{K}}, \boldsymbol{\mu}_{h_{K}}^{N_{K}}, \partial_{t} \mathbf{c}_{h_{K}}^{N_{K}}$ and $\mathbf{u}_{h_{K}}^{N_{K}}$ are respectively bounded in the following norm: $\mathrm{L}^{\infty}\left(0, t_{f},\left(\mathrm{H}^{1}(\Omega)\right)^{3}\right), \mathrm{L}^{2}\left(0, t_{f},\left(\mathrm{H}^{1}(\Omega)\right)^{3}\right), \mathrm{L}^{2}\left(0, t_{f},\left(\mathrm{H}^{1}(\Omega)\right)^{\prime}\right)$, $\mathrm{L}^{2}\left(0, t_{f},\left(\mathrm{H}^{1}(\Omega)\right)^{d}\right)$.

The weak convergences we write above are not sufficient to pass to the limit in the non linear terms of the Cahn-Hilliard and Navier-Stokes systems. We prove in the next two propositions that it is possible to obtain strong convergence for order parameters and velocity in some suitable function spaces.

Proposition 5.5. Up to subsequences, we have the following convergences when $K \rightarrow+\infty$ :

$$
\begin{aligned}
& \mathbf{c}_{h_{K}}^{N_{K}} \rightarrow \mathbf{c} \text { in } \mathcal{C}^{0}\left(0, t_{f},\left(\mathrm{~L}^{q}(\Omega)\right)^{3}\right) \text { strong, for all } 1 \leqslant q<+\infty \text { for } d=2 \text {, } \\
& \text { or } 1 \leqslant q<6 \text { for } d=3 \text {, } \\
& \mathbf{c}_{h_{K}}^{N_{K}} \rightarrow \mathbf{c}, \quad \underline{\mathbf{c}}_{h_{K}}^{N_{K}} \rightarrow \mathbf{c}, \quad \overline{\mathbf{c}}_{h_{K}}^{N_{K}} \rightarrow \mathbf{c} \quad \text { in } \mathrm{L}^{2}\left(0, t_{f},\left(\mathrm{~L}^{2}(\Omega)\right)^{3}\right) \text { strong. }
\end{aligned}
$$

Proof. The sequence $\mathbf{c}_{h_{K}}^{N_{K}}$ is bounded in $\mathrm{L}^{\infty}\left(0, t_{f},\left(\mathrm{H}^{1}(\Omega)\right)^{3}\right)$ and its time derivative $\partial_{t} \mathbf{c}_{h_{K}}^{N_{K}}$ is bounded in $\mathrm{L}^{2}\left(0, t_{f},\left(\mathrm{H}^{1}(\Omega)\right)^{\prime}\right)$. We obtain the strong convergence $(5.16)$ of order parameters by applying the Aubin-Lions-Simon compactness theorem [24]. From this convergence and using the inequality (5.5), we deduce the strong convergences (5.17).

To prove the result of strong convergence on the velocity, we need to apply a more precise compactness result since we do not have any estimate on its time derivative. We apply a compactness theorem due to Simon in which the condition on the time derivative is replaced by an estimation on time translates.

First, we write the term to estimate. This term is defined from the discrete function $\underline{\mathbf{u}}_{h}^{N}$ which is piecewise constant (in time) and its time translate. We link this term to the values $\mathbf{u}_{h}^{n}$ of the function on each time intervals in order to exploit estimates proved in section 5.1. To simplify the notation, we omit in this lemma, the index $K$ in the notation $h_{K}$ and $N_{K}$.

Lemma 5.6. Let $\tau \in] 0, t_{f}[$. We denote by $i \in \llbracket 0 ; N-1 \rrbracket$ the unique index such that $t^{i} \leqslant \tau<t^{i+1}$. Then, we have:

(i) if $\tau<\Delta t$ then

$$
\int_{0}^{t_{f}-\tau}\left|\underline{\mathbf{u}}_{h}^{N}(s+\tau, \cdot)-\underline{\mathbf{u}}_{h}^{N}(s, \cdot)\right|_{\left(\mathrm{L}^{2}(\Omega)\right)^{d}}^{2} d s=\tau \sum_{n=0}^{N-2}\left|\mathbf{u}_{h}^{n+1}-\mathbf{u}_{h}^{n}\right|_{\left(\mathrm{L}^{2}(\Omega)\right)^{d}}^{2}
$$

(ii) in all cases, we have:

$$
\begin{aligned}
\int_{0}^{t_{f}-\tau} & \left|\underline{\mathbf{u}}_{h}^{N}(s+\tau, \cdot)-\underline{\mathbf{u}}_{h}^{N}(s, \cdot)\right|_{\left(\mathrm{L}^{2}(\Omega)\right)^{d}}^{2} d s \\
& \leqslant \sum_{n=0}^{N-i-1} \Delta t\left|\mathbf{u}_{h}^{n+i}-\mathbf{u}_{h}^{n}\right|_{\left(\mathrm{L}^{2}(\Omega)\right)^{d}}^{2}+\sum_{n=0}^{N-i-2} \Delta t\left|\mathbf{u}_{h}^{n+i+1}-\mathbf{u}_{h}^{n}\right|_{\left(\mathrm{L}^{2}(\Omega)\right)^{d}}^{2}
\end{aligned}
$$

We can now state the proposition giving the strong convergence for the velocity. 
Proposition 5.7. Up to subsequences, we have the following convergences when $K \rightarrow+\infty$ :

$$
\mathbf{u}_{h_{K}}^{N_{K}} \rightarrow \mathbf{u}, \quad \underline{\mathbf{u}}_{h_{K}}^{N_{K}} \rightarrow \mathbf{u}, \quad \overline{\mathbf{u}}_{h_{K}}^{N_{K}} \rightarrow \mathbf{u}, \quad \text { in } \mathrm{L}^{2}\left(0, t_{f},\left(\mathrm{~L}^{2}(\Omega)\right)^{d}\right) \text { strong. }
$$

Proof. The proof relies on a compactness theorem due to Simon [24, Theorem 5, p.84] which state that the embedding

$$
\mathrm{L}^{2}(] 0, t_{f}\left[,\left(\mathrm{H}^{1}(\Omega)\right)^{d}\right) \cap \mathrm{N}_{2}^{\frac{1}{8}}(] 0, t_{f}\left[,\left(\mathrm{~L}^{2}(\Omega)\right)^{d}\right) \hookrightarrow \mathrm{L}^{2}(] 0, t_{f}\left[,\left(\mathrm{~L}^{2}(\Omega)\right)^{d}\right)
$$

is compact. The Nikolskii space $\mathrm{N}_{2}^{\frac{1}{8}}(] 0, t_{f}\left[,\left(\mathrm{~L}^{2}(\Omega)\right)^{d}\right)$ is defined by:

$$
\begin{aligned}
\mathrm{N}_{2}^{\frac{1}{8}}(] 0, t_{f}\left[,\left(\mathrm{~L}^{2}(\Omega)\right)^{d}\right)= & \left\{\mathbf{v} \in \mathrm{L}^{2}(] 0, t_{f}\left[,\left(\mathrm{~L}^{2}(\Omega)\right)^{d}\right) ;\right. \\
& \exists C>0, \forall \tau \in] 0, t_{f}\left[,|\mathbf{v}(\cdot+\tau, \cdot)-\mathbf{v}|_{\mathrm{L}^{2}(] 0, t_{f}-\tau\left[,\left(\mathrm{L}^{2}(\Omega)\right)^{d}\right)} \leqslant C \tau^{\frac{1}{8}}\right\},
\end{aligned}
$$

with the norm

$$
\begin{aligned}
|\mathbf{v}|_{\mathrm{N}_{2}^{\frac{1}{8}}(] 0, t_{f}\left[,\left(\mathrm{~L}^{2}(\Omega)\right)^{d}\right)}= & \left(|\mathbf{v}|_{\mathrm{L}^{2}(] 0, t_{f}\left[,\left(\mathrm{~L}^{2}(\Omega)\right)^{d}\right)}^{2}\right. \\
& \left.\quad+\sup _{0<\tau<t_{f}}\left(\frac{1}{\tau^{\frac{1}{8}}}|\mathbf{v}(\cdot+\tau, \cdot)-\mathbf{v}|_{\mathrm{L}^{2}(] 0, t_{f}-\tau\left[,\left(\mathrm{L}^{2}(\Omega)\right)^{d}\right)}\right)^{2}\right)^{\frac{1}{2}} .
\end{aligned}
$$

Thus, since the sequence $\underline{\mathbf{u}}_{h_{K}}^{N_{K}}$ is bounded in the spaces $\mathrm{L}^{2}(] 0, t_{f}\left[,\left(\mathrm{H}^{1}(\Omega)\right)^{d}\right)$ and $\mathrm{L}^{2}(] 0, t_{f}\left[,\left(\mathrm{~L}^{2}(\Omega)\right)^{d}\right)$ (cf equations (5.3) and (5.4)), it is sufficient to prove that it is bounded in the space $\mathrm{N}_{2}^{\frac{1}{8}}(] 0, t_{f}\left[,\left(\mathrm{~L}^{2}(\Omega)\right)^{d}\right)$, to obtain the conclusion. Let $\left.\tau \in\right] 0, t_{f}[$. We still omit the index $K$ in the notation $h_{K}$ and $N_{K}$.

(i) If $\tau<\Delta t$ then owing to lemma 5.6, we have:

$$
\int_{0}^{t_{f}-\tau}\left|\underline{\mathbf{u}}_{h}^{N}(s+\tau, \cdot)-\underline{\mathbf{u}}_{h}^{N}(s, \cdot)\right|_{\left(\mathrm{L}^{2}(\Omega)\right)^{d}}^{2} d s=\tau \sum_{n=0}^{N-2}\left|\mathbf{u}_{h}^{n+1}-\mathbf{u}_{h}^{n}\right|_{\left(\mathrm{L}^{2}(\Omega)\right)^{d}}^{2} \leqslant K_{3} \tau .
$$

(ii) If $\tau \geqslant \Delta t$ then owing to lemma 5.6, and then using the inequality (5.7), we have:

$$
\begin{aligned}
\int_{0}^{t_{f}-\tau}\left|\underline{\mathbf{u}}_{h}^{N}(s+\tau, \cdot)-\underline{\mathbf{u}}_{h}^{N}(s, \cdot)\right|_{\left(\mathrm{L}^{2}(\Omega)\right)^{d}}^{2} d s & \\
& \leqslant \sum_{n=0}^{N-i-1} \Delta t\left|\mathbf{u}_{h}^{n+i}-\mathbf{u}_{h}^{n}\right|_{\left(\mathrm{L}^{2}(\Omega)\right)^{d}}^{2}+\sum_{n=0}^{N-i-2} \Delta t\left|\mathbf{u}_{h}^{n+i+1}-\mathbf{u}_{h}^{n}\right|_{\left(\mathrm{L}^{2}(\Omega)\right)^{d}}^{2} \\
& \leqslant K_{5}\left[\left(t^{i}\right)^{\frac{1}{4}}+\left(t^{i+1}\right)^{\frac{1}{4}}\right] \leqslant K_{5}\left[1+2^{\frac{1}{4}}\right] \tau^{\frac{1}{4}},
\end{aligned}
$$

since we have $t^{i} \leqslant \tau$ and $t^{i+1}=t^{i}+\Delta t \leqslant 2 \tau$.

In all cases, we obtained the existence of a positive constant $K_{6}$ (independent of $h$ and $\Delta t)$ such that:

$$
\left.\int_{0}^{t_{f}-\tau}\left|\underline{\mathbf{u}}_{h}^{N}(s+\tau, \cdot)-\underline{\mathbf{u}}_{h}^{N}(s, \cdot)\right|_{\left(\mathrm{L}^{2}(\Omega)\right)^{d}}^{2} d s \leqslant K_{6} \tau^{\frac{1}{4}}, \quad \forall \tau \in\right] 0, t_{f}[.
$$

This concludes the proof of the convergence of $\underline{\mathbf{u}}_{h}^{N}$. The convergences of $\mathbf{u}_{h_{K}}^{N_{K}}$ and $\overline{\mathbf{u}}_{h_{K}}^{N_{K}}$ are then obtained thanks to the inequality (5.5). 
5.3. Passing to the limit in the scheme. The convergences obtained in section 5.2 allow to pass to the limit in the discrete system.

For the Cahn-Hilliard system (without the transport term), this work was already done in details in reference [7]. We focus here on the transport term and on the Navier-Stokes equation.

To simplify the notation, we still omit the index $K$ in the notation $N_{K}$ and $h_{K}$ but when we say "convergence" it means $K \rightarrow+\infty$ (and consequently $N_{K} \rightarrow+\infty$ and $\left.h_{K} \rightarrow 0\right)$.

5.3.1. Transport term in the Cahn-Hilliard equation. Let $\nu^{\mu} \in \mathcal{C}^{\infty}(\bar{\Omega})$ a given function and $\tau \in \mathcal{C}_{c}^{\infty}(] 0, t_{f}[)$. We define $\nu_{h}^{\mu}$ as the $\mathrm{H}^{1}$ projection of the function $\nu^{\mu}$ on $\mathcal{V}_{h}^{\mu}$. We have to prove the following convergence:

$$
\begin{aligned}
\int_{0}^{t_{f}} \int_{\Omega}\left[\underline{c}_{i h}^{N}-\alpha_{i}\right]\left[\underline{\mathbf{u}}_{h}^{N}-\frac{\Delta t}{\varrho_{0}} \sum_{j=1}^{3}\left(\underline{c}_{j h}^{N}\right.\right. & \left.\left.-\alpha_{j}\right) \nabla \mu_{j h}^{N}\right] \cdot \nabla \nu_{h}^{\mu} d x \tau(t) d t \\
& \longrightarrow \int_{0}^{t_{f}} \int_{\Omega}\left(c_{i}-\alpha_{i}\right) \mathbf{u} \cdot \nabla \nu^{\mu} d x \tau(t) d t .
\end{aligned}
$$

We proceed in two steps, separately considering two terms of the left hand side: the standard transport term and the additional term which ensures the unconditional stability.

The following inequalities allows to identify the limit of the first term:

$$
\begin{aligned}
& \left|\int_{0}^{t_{f}} \int_{\Omega}\left(c_{i h}^{N}-\alpha_{i}\right) \underline{\mathbf{u}}_{h}^{N} \cdot \nabla \nu_{h}^{\mu} d x \tau(t) d t-\int_{0}^{t_{f}} \int_{\Omega}\left(c_{i}-\alpha_{i}\right) \mathbf{u} \cdot \nabla \nu^{\mu} d x \tau(t) d t\right| \\
& \quad \leqslant|\tau|_{L^{\infty}\left(0, t_{f}\right)}\left|\nu_{h}^{\mu}-\nu^{\mu}\right|_{\mathrm{H}^{1}(\Omega)}\left|\underline{c}_{i h}^{N}-\alpha_{i}\right|_{\mathrm{L}^{2}\left(0, t_{f}, \mathrm{H}^{1}(\Omega)\right)}\left|\underline{\mathbf{u}}_{h}^{N}\right|_{\mathrm{L}^{2}\left(0, t_{f},\left(\mathrm{H}^{1}(\Omega)\right)^{d}\right)} \\
& \quad+|\tau|_{\mathrm{L}^{\infty}\left(0, t_{f}\right)}\left|\nabla \nu^{\mu}\right|_{\mathrm{L}^{3}(\Omega)}\left|\underline{c}_{i h}^{N}-\alpha_{i}\right|_{\mathrm{L}^{2}\left(0, t_{f}, \mathrm{H}^{1}(\Omega)\right)}\left|\underline{\mathbf{u}}_{h}^{N}-\mathbf{u}\right|_{\mathrm{L}^{2}\left(0, t_{f},\left(\mathrm{~L}^{2}(\Omega)\right)^{d}\right)} \\
& \quad+|\tau|_{\mathrm{L}^{\infty}\left(0, t_{f}\right)}\left|\nabla \nu^{\mu}\right|_{\mathrm{L}^{3}(\Omega)}\left|\underline{c}_{i h}^{N}-c_{i}\right|_{\mathrm{L}^{2}\left(0, t_{f}, \mathrm{~L}^{2}(\Omega)\right)}|\mathbf{u}|_{\mathrm{L}^{2}\left(0, t_{f},\left(\mathrm{H}^{1}(\Omega)\right)^{d}\right)} \\
& \quad \longrightarrow 0
\end{aligned}
$$

since $\underline{c}_{i h}^{N}$ is bounded in $\mathrm{L}^{2}\left(0, t_{f}, \mathrm{H}^{1}(\Omega)\right), \underline{\mathbf{u}}_{h}^{N}$ is bounded in $\mathrm{L}^{2}\left(0, t_{f},\left(\mathrm{H}^{1}(\Omega)\right)^{d}\right), \underline{c}_{i h}^{N}$ (strongly) converges in $\mathrm{L}^{2}\left(0, t_{f}, \mathrm{~L}^{2}(\Omega)\right)$ towards $c_{i}$ (cf equation (5.17)), $\underline{\mathbf{u}}_{h}^{N}$ (strongly) converges in $\mathrm{L}^{2}\left(0, t_{f},\left(\mathrm{~L}^{2}(\Omega)\right)^{d}\right)$ towards $\mathbf{u}(c f$ equation (5.18)) and, owing to assumption (2.5), $\left|\nu^{\mu}-\nu_{h}^{\mu}\right|_{\mathrm{H}^{1}(\Omega)}=\inf _{\nu_{h} \in \mathcal{V}^{\mu}}\left|\nu^{\mu}-\nu_{h}\right|_{\mathrm{H}^{1}(\Omega)} \underset{h \rightarrow 0}{\longrightarrow} 0$.

We now use the fact than the sequences $\underline{c}_{i h}^{N}$ are $\mu_{j h}^{N}$ are respectively bounded in $\mathrm{L}^{\infty}\left(0, t_{f}, \mathrm{H}^{1}(\Omega)\right)$ and $\mathrm{L}^{2}\left(0, t_{f}, \mathrm{H}^{1}(\Omega)\right)$ norm, the inverse inequality (2.8) and the condition (5.2) on the sequences $h_{K}$ and $N_{K}$ to show that the second term convergences towards 0 :

$$
\begin{aligned}
& \left|\int_{0}^{t_{f}} \int_{\Omega}\left[\underline{c}_{i h}^{N}-\alpha_{i}\right]\left[\frac{\Delta t}{\varrho_{0}} \sum_{j=1}^{3}\left(\underline{c}_{j h}^{N}-\alpha_{j}\right) \nabla \mu_{j h}^{N}\right] \cdot \nabla \nu_{h}^{\mu} d x \tau(t) d t\right| \\
& \quad+\frac{\Delta t}{\varrho_{0}}|\tau|_{\mathrm{L}^{2}}\left|\nabla \nu^{\mu}\right|_{\mathrm{L}^{\infty}}\left|\underline{c}_{i h}^{N}-\alpha_{i}\right|_{\mathrm{L}^{\infty}\left(\mathrm{H}^{1}\right)} \sum_{j=1}^{3}\left|\underline{c}_{j h}^{N}-\alpha_{j}\right|_{\mathrm{L}^{\infty}\left(\mathrm{H}^{1}\right)}\left|\mu_{j h}^{N}\right|_{\mathrm{L}^{2}\left(\mathrm{H}^{1}\right)} \\
& \longrightarrow 0
\end{aligned}
$$


Thus, we proved that the convergence (5.19) holds. Re-using (exactly as it is) the reasoning presented in [7] allows to pass to the limit in the other terms of the Cahn-Hilliard system.

5.3.2. Navier-Stokes system. Let $\boldsymbol{\nu}^{\mathbf{u}} \in \mathcal{C}_{c}^{\infty}(\Omega)$ satisfying $\operatorname{div}\left(\boldsymbol{\nu}^{\mathbf{u}}\right)=0$ and $\tau \in \mathcal{C}^{1}\left(\left[0, t_{f}\right]\right)$ such that $\tau\left(t_{f}\right)=0$.

We introduce the space

$$
Z_{h}=\left\{\mathbf{z}_{h} \in \mathcal{V}_{h, 0}^{\mathbf{u}} ; \quad \forall \nu_{h}^{p} \in \mathcal{V}_{h}^{p}, \int_{\Omega} \operatorname{div}\left(\mathbf{z}_{h}\right) \nu_{h}^{p} d x=0\right\}
$$

The inf-sup condition (2.9) implies that the function $\boldsymbol{\nu}^{\mathbf{u}} \in \mathrm{H}_{0}^{1}(\Omega)$ which is divergence free can be "well approximated" with functions in $Z_{h}$. This is detailed in the proposition 5.8 .

Proposition 5.8 (Approximation of divergence free functions, [8, eq. 12.5.17]). We have the following inequality:

$$
\inf _{\mathbf{z}_{h} \in Z_{h}}\left|\boldsymbol{\nu}^{\mathbf{u}}-\mathbf{z}_{h}\right|_{\mathrm{H}^{1}(\Omega)} \leqslant \frac{1}{\Theta} \inf _{\nu_{h}^{\mathbf{u}} \in \mathcal{V}_{h, 0}^{\mathbf{u}}}\left|\boldsymbol{\nu}^{\mathbf{u}}-\boldsymbol{\nu}_{h}^{\mathbf{u}}\right|_{\mathrm{H}^{1}(\Omega)} .
$$

Let $\boldsymbol{\nu}_{h}^{\mathbf{u}}$ be the $\mathrm{H}^{1}$ projection of $\boldsymbol{\nu}^{\mathbf{u}}$ on the space $Z_{h}$. The proposition 5.8 and the assumption (2.6) show that

$$
\boldsymbol{\nu}_{h}^{\mathbf{u}} \rightarrow \boldsymbol{\nu}^{\mathbf{u}}, \quad \text { in }\left(\mathrm{H}^{1}(\Omega)\right)^{d} \text { strong. }
$$

We use $\boldsymbol{\nu}_{h}^{\mathbf{u}}$ as a test function in the first equation of (2.11). We then multiply by $\tau(t), t \in] t^{n}, t^{n+1}$ [, integrate between $t^{n}$ and $t^{n+1}$, and sum up for $n$ from 0 to $N-1$ so that we rebuilt a variational formulation on $] 0, t_{f}[\times \Omega$. The unsteady term is modified by a discrete integration by part:

$$
\begin{aligned}
\sum_{n=0}^{N-1} \int_{t^{n}}^{t^{n+1}} \int_{\Omega} \varrho_{0} \frac{\mathbf{u}_{h}^{n+1}-\mathbf{u}_{h}^{n}}{\Delta t} \cdot \boldsymbol{\nu}_{h}^{\mathbf{u}} d x \tau(t) d t= & -\varrho_{0} \int_{0}^{t_{f}} \int_{\Omega} \underline{\mathbf{u}}_{h}^{N}(t, x) \cdot \boldsymbol{\nu}_{h}^{\mathbf{u}} d x \frac{\tau(t)-\tau(t-\Delta t)}{\Delta t} d t \\
& +\varrho_{0} \int_{\Omega} \mathbf{u}_{h}^{n=N}(x) \cdot \boldsymbol{\nu}_{h}^{\mathbf{u}}(x) d x \int_{0}^{1} \tau\left(t_{f}-t \Delta t\right) d t \\
& -\varrho_{0} \int_{\Omega} \mathbf{u}_{h}^{0}(x) \cdot \boldsymbol{\nu}_{h}^{\mathbf{u}}(x) d x \int_{0}^{1} \tau(\Delta t(t-1)) d t
\end{aligned}
$$

Thus, we obtain the following formulation of the scheme in which we can pass to the limit:

$$
\begin{aligned}
& -\underbrace{\varrho_{0} \int_{0}^{t_{f}} \int_{\Omega} \underline{\mathbf{u}}_{h}^{N}(t, x) \cdot \boldsymbol{\nu}_{h}^{\mathbf{u}} d x \frac{\tau(t)-\tau(t-\Delta t)}{\Delta t} d t}_{T_{1}}-\underbrace{\varrho_{0} \int_{\Omega} \mathbf{u}_{h}^{0}(x) \cdot \boldsymbol{\nu}_{h}^{\mathbf{u}}(x) d x \int_{0}^{1} \tau(\Delta t(t-1)) d t}_{T_{2}} \\
& +\underbrace{\frac{1}{2} \int_{0}^{t_{f}} \int_{\Omega} \varrho_{0}\left(\underline{\mathbf{u}}_{h}^{N} \cdot \nabla\right) \overline{\mathbf{u}}_{h}^{N} \cdot \boldsymbol{\nu}_{h}^{\mathbf{u}} d x \tau(t) d t}_{T_{3}}-\underbrace{\frac{1}{2} \int_{0}^{t_{f}} \int_{\Omega} \varrho_{0}\left(\underline{\mathbf{u}}_{h}^{N} \cdot \nabla\right) \boldsymbol{\nu}_{h}^{\mathbf{u}} \cdot \overline{\mathbf{u}}_{h}^{N} d x \tau(t) d t}_{T_{4}} \\
& +\underbrace{\int_{0}^{t_{f}} \int_{\Omega} 2 \eta\left(\overline{\mathbf{c}}_{h}^{N}\right) D \overline{\mathbf{u}}_{h}^{N}: D \boldsymbol{\nu}_{h}^{\mathbf{u}} d x \tau(t) d t}_{T_{5}}=\underbrace{\int_{0}^{t_{f}} \int_{\Omega} \varrho_{0} \mathbf{g} \cdot \boldsymbol{\nu}_{h}^{\mathbf{u}} d x \tau(t) d t}_{T_{6}} \\
& -\underbrace{\int_{0}^{t_{f}} \int_{\Omega} \sum_{j=1}^{3}\left(\underline{c}_{j h}^{N}-\alpha_{j}\right) \nabla \mu_{j h}^{N} \cdot \boldsymbol{\nu}_{h}^{\mathbf{u}} d x \tau(t) d t}_{T_{7}}-\underbrace{\varrho_{0} \int_{\Omega} \mathbf{u}_{h}^{n=N}(x) \cdot \boldsymbol{\nu}_{h}^{\mathbf{u}}(x) d x \int_{0}^{1} \tau\left(t_{f}-t \Delta t\right) d t}_{T_{8}} .
\end{aligned}
$$


The limit of the term $T_{1}$ is readily obtained from strong convergences (5.18), (5.20) and those of functions $t \mapsto \frac{\tau(t)-\tau(t-\Delta t)}{\Delta t}$ towards $\tau^{\prime}$ in $\mathrm{L}^{2}\left(0, t_{f}\right)$ (obtained for instance with dominated convergence theorem since the function $\tau$ is in $\left.\mathcal{C}^{1}\left(\left[0, t_{f}\right]\right)\right)$ :

$$
T_{1} \longrightarrow \varrho_{0} \int_{0}^{t_{f}} \int_{\Omega} \mathbf{u} \cdot \boldsymbol{\nu}^{\mathbf{u}} d x \tau^{\prime}(t) d t .
$$

The term $T_{2}$ allows to show that $\mathbf{u}$ satisfies the initial condition (1.11) in a weak sense. The convergences $(2.10),(5.20)$ and the uniform convergence on $\left[0, t_{f}\right]$ of the function $t \mapsto \tau(\Delta t(t-1))$ towards the constant function equal to $\tau(0)$ yields:

$$
T_{2} \rightarrow \varrho_{0} \int_{\Omega} \mathbf{u}^{0}(x) \cdot \boldsymbol{\nu}^{\mathbf{u}}(x) d x \tau(0) d t .
$$

Concerning the term $T_{3}$, the following inequality allows to conclude:

$$
\begin{aligned}
& \left|\int_{0}^{t_{f}} \int_{\Omega}\left(\underline{\mathbf{u}}_{h}^{N} \cdot \nabla\right) \overline{\mathbf{u}}_{h}^{N} \cdot \boldsymbol{\nu}_{h}^{\mathbf{u}} d x \tau(t) d t-\int_{0}^{t_{f}} \int_{\Omega}(\mathbf{u} \cdot \nabla) \mathbf{u} \cdot \boldsymbol{\nu}^{\mathbf{u}} d x \tau(t) d t\right| \\
& \leqslant|\tau|_{L^{\infty}}\left[\left|\overline{\mathbf{u}}_{h}^{N}\right|_{\mathrm{L}^{2}\left(\left(\mathrm{H}^{1}\right)^{d}\right)}\left|\boldsymbol{\nu}_{h}^{\mathbf{u}}-\boldsymbol{\nu}^{\mathbf{u}}\right|_{\mathrm{L}^{4}}\left|\underline{\mathbf{u}}_{h}^{N}\right|_{\mathrm{L}^{2}\left(\left(\mathrm{~L}^{4}\right)^{d}\right)}+\left|\underline{\mathbf{u}}_{h}^{N}-\mathbf{u}\right|_{\mathrm{L}^{2}\left(\left(\mathrm{~L}^{2}\right)^{d}\right)}\left|\overline{\mathbf{u}}_{h}^{N}\right|_{\mathrm{L}^{2}\left(\left(\mathrm{H}^{1}\right)^{d}\right)}\left|\boldsymbol{\nu}^{\mathbf{u}}\right|_{\left(\mathrm{L}^{\infty}\right)^{d}}\right] \\
& +\left|\int_{0}^{t_{f}} \int_{\Omega}(\mathbf{u} \cdot \nabla)\left(\overline{\mathbf{u}}_{h}^{N}-\mathbf{u}\right) \cdot \boldsymbol{\nu}^{\mathbf{u}} d x \tau(t) d t\right| \\
& \longrightarrow 0 \text {. }
\end{aligned}
$$

Indeed, since the sequences $\left(\underline{\mathbf{u}}_{h}^{N}\right)$ and $\left(\overline{\mathbf{u}}_{h}^{N}\right)$ are bounded in $\mathrm{L}^{2}\left(0, t_{f},\left(\mathrm{H}^{1}(\Omega)\right)^{d}\right)$, the convergences (5.18) and (5.20) show that the first two terms of the above right hand side tend to 0 . And the last one (the term involving the integral), it also tends to 0 by weak convergence of $\nabla \overline{\mathbf{u}}_{h}^{N}$ towards $\nabla \mathbf{u}$ in $\mathrm{L}^{2}\left(0, t_{f},\left(\mathrm{~L}^{2}(\Omega)\right)^{d}\right)$ (a component-bycomponent reasoning gives the result since for all $1 \leqslant i, j \leqslant d$, the function $(t, x) \mapsto$ $\mathbf{u}_{i}(x) \boldsymbol{\nu}_{j}^{\mathbf{u}}(x) \tau(t)$ lies in $\left.\mathrm{L}^{2}\left(0, t_{f}, \mathrm{~L}^{2}(\Omega)\right)\right)$.

The term $T_{4}$ is treated in the same way:

$$
\begin{aligned}
& \left|\int_{0}^{t_{f}} \int_{\Omega}\left(\underline{\mathbf{u}}_{h}^{N} \cdot \nabla\right) \boldsymbol{\nu}_{h}^{\mathbf{u}} \cdot \overline{\mathbf{u}}_{h}^{N} d x \tau(t) d t-\int_{0}^{t_{f}} \int_{\Omega}(\mathbf{u} \cdot \nabla) \boldsymbol{\nu}^{\mathbf{u}} \cdot \mathbf{u} d x \tau(t) d t\right| \\
& \leqslant|\tau|_{\mathrm{L}^{\infty}}\left[\left|\underline{\mathbf{u}}_{h}^{N}\right|_{\mathrm{L}^{2}\left(\left(\mathrm{~L}^{4}\right)^{d}\right)}\left|\boldsymbol{\nu}_{h}^{\mathbf{u}}-\boldsymbol{\nu}^{\mathbf{u}}\right|_{\mathrm{H}^{1}}\left|\overline{\mathbf{u}}_{h}^{N}\right|_{\mathrm{L}^{2}\left(\left(\mathrm{~L}^{4}\right)^{d}\right)}+\left|\underline{\mathbf{u}}_{h}^{N}\right|_{\mathrm{L}^{2}\left(\left(\mathrm{~L}^{6}\right)^{d}\right)}\left|\nabla \boldsymbol{\nu}^{\mathbf{u}}\right|_{\left(\mathrm{L}^{3}\right)^{d}}\left|\overline{\mathbf{u}}_{h}^{N}-\mathbf{u}\right|_{\mathrm{L}^{2}\left(\left(\mathrm{~L}^{2}\right)^{d}\right)}\right] \\
& +\left|\underline{\mathbf{u}}_{h}^{N}-\mathbf{u}\right|_{\mathrm{L}^{2}\left(0, t_{f},\left(\mathrm{~L}^{2}\right)^{d}\right)}\left|\nabla \boldsymbol{\nu}^{\mathbf{u}}\right|_{\left(\mathrm{L}^{3}\right)^{d}}|\mathbf{u}|_{\mathrm{L}^{2}\left(\left(\mathrm{~L}^{6}\right)^{d}\right)}|\tau(t)|_{\mathrm{L}^{\infty}},
\end{aligned}
$$

the conclusion being now obtained using the convergences (5.18), (5.20) and the fact that the two sequences $\underline{\mathbf{u}}_{h}^{N}$ and $\overline{\mathbf{u}}_{h}^{N}$ are bounded in $\mathrm{L}^{2}\left(0, t_{f},\left(\mathrm{H}^{1}(\Omega)\right)^{d}\right)$.

The limit of the term (5) is obtained using the following convergence (up to a subsequence):

$$
\eta\left(\overline{\mathbf{c}}_{h_{K}}^{N}\right) \rightarrow \eta(\mathbf{c}) \quad \text { in } \mathrm{L}^{2}\left(0, t_{f},\left(\mathrm{~L}^{2}(\Omega)\right)^{d}\right) \text { strong. }
$$

This convergence is proved by using the dominated convergence theorem (the viscosity $\eta$ is a bounded continuous function and $\overline{\mathbf{c}}_{h_{K}}^{N}$ strongly converge in $\mathrm{L}^{2}\left(0, t_{f},\left(\mathrm{~L}^{2}(\Omega)\right)^{3}\right)$, almost everywhere up to a subsequence). 
Thus, using (5.20), (5.21), the fact that the sequence $\overline{\mathbf{u}}_{h}^{N}$ is bounded in $\mathrm{L}^{2}\left(0, t_{f},\left(\mathrm{H}^{1}(\Omega)\right)^{d}\right)$, and the weak convergence of $D \overline{\mathbf{u}}_{h}^{N}$ towards $D \mathbf{u}$ in $\mathrm{L}^{2}\left(0, t_{f},\left(\mathrm{~L}^{2}(\Omega)\right)^{d}\right)$, we obtain

$$
\begin{aligned}
& \left|\int_{0}^{t_{f}} \int_{\Omega} 2 \eta\left(\overline{\mathbf{c}}_{h_{K}}^{N}\right) D \overline{\mathbf{u}}_{h}^{N}: D \boldsymbol{\nu}_{h}^{\mathbf{u}} d x \tau(t) d t-\int_{0}^{t_{f}} \int_{\Omega} 2 \eta(\mathbf{c}) D \mathbf{u}: D \boldsymbol{\nu}^{\mathbf{u}} d x \tau(t) d t\right| \\
& \leqslant\left|\overline{\mathbf{u}}_{h}^{N}\right|_{\mathrm{L}^{2}\left(\left(\mathrm{H}^{1}\right)^{d}\right)}\left[2 \eta_{\max }\left|\boldsymbol{\nu}_{h}^{\mathbf{u}}-\boldsymbol{\nu}^{\mathbf{u}}\right|_{\left(\mathrm{H}^{1}\right)^{d}}|\tau|_{\mathrm{L}^{2}}+2\left|\eta\left(\overline{\mathbf{c}}_{h_{K}}^{N}\right)-\eta(\mathbf{c})\right|_{\mathrm{L}^{2}\left(0, t_{f}, \mathrm{~L}^{2}\right)}\left|\nabla \boldsymbol{\nu}^{\mathbf{u}}\right|_{\mathrm{L}^{\infty}(\Omega)}|\tau|_{\mathrm{L}^{\infty}}\right] \\
& \quad+\left|\int_{0}^{t_{f}} \int_{\Omega} 2 \eta(\mathbf{c}) D\left(\overline{\mathbf{u}}_{h}^{N}-\mathbf{u}\right): D \boldsymbol{\nu}^{\mathbf{u}} d x \tau(t) d t\right|
\end{aligned}
$$

$\longrightarrow 0$.

By (5.20), the convergence of term $T_{6}$ is straightforward:

$$
T_{6} \rightarrow \int_{\Omega} \varrho_{0} \mathbf{g} \cdot \boldsymbol{\nu}^{\mathbf{u}} d x \tau(t) d t
$$

The convergence of the capillary forces term $T_{7}$ is obtained as follows:

$$
\begin{aligned}
& \left|\int_{0}^{t_{f}} \int_{\Omega} \sum_{j=1}^{3}\left(\underline{c}_{j h}^{N}-\alpha_{j}\right) \nabla \mu_{j h}^{N} \cdot \boldsymbol{\nu}_{h}^{\mathbf{u}} d x \tau(t) d t-\int_{0}^{t_{f}} \int_{\Omega} \sum_{j=1}^{3}\left(c_{j h}-\alpha_{j}\right) \nabla \mu_{j h} \cdot \boldsymbol{\nu}^{\mathbf{u}} d x \tau(t) d t\right| \\
& \leqslant \sum_{j=1}^{3}\left|\nabla \mu_{j h}^{N}\right|_{\mathrm{L}^{2}\left(\left(\mathrm{~L}^{2}\right)^{d}\right)}\left[\left|\underline{c}_{j h}^{N}-\alpha_{j}\right|_{\mathrm{L}^{\infty}\left(\mathrm{L}^{4}\right)}\left|\boldsymbol{\nu}_{h}^{\mathbf{u}}-\boldsymbol{\nu}^{\mathbf{u}}\right|_{\mathrm{L}^{4}}|\tau|_{\mathrm{L}^{2}}+\left|\underline{c}_{j h}^{N}-c_{j h}\right|_{\mathrm{L}^{2}\left(\left(\mathrm{~L}^{2}\right)^{d}\right)}\left|\boldsymbol{\nu}^{\mathbf{u}}\right|_{\mathrm{L}^{\infty}}|\tau|_{\mathrm{L}^{\infty}}\right] \\
& \quad+\left|\int_{\Omega} \sum_{j=1}^{3}\left(c_{j h}-\alpha_{j}\right) \nabla\left(\mu_{j h}^{N}-\mu_{j}\right) \cdot \boldsymbol{\nu}^{\mathbf{u}} d x \tau(t) d t\right|
\end{aligned}
$$

$\longrightarrow 0$.

The first two terms of the right hand side tend to 0 thanks to convergences (5.17) and (5.20) since the sequences $\left(\underline{c}_{j h}^{N}\right)$ and $\left(\mu_{j h}^{N}\right)$ are bounded in $\mathrm{L}^{2}\left(0, t_{f}, \mathrm{H}^{1}(\Omega)\right)$ and $\mathrm{L}^{\infty}\left(0, t_{f}, \mathrm{H}^{1}(\Omega)\right)$ respectively. The last term tends to 0 by weak convergence of $\nabla \mu_{j h}^{N}$ towards $\nabla \mu_{j}$ in $\mathrm{L}^{2}\left(0, t_{f},\left(\mathrm{~L}^{2}(\Omega)\right)^{d}\right)$.

Finally, it only remains to prove that the residual term $T_{8}$ tends to 0 . This simply comes from the fact that:

$$
\left|\int_{\Omega} \mathbf{u}_{h}^{N}(x) \cdot \boldsymbol{\nu}_{h}^{\mathbf{u}}(x) d x\right| \leqslant\left|\mathbf{u}_{h}^{N}(\cdot)\right|_{\mathrm{L}^{2}(\Omega)}\left|\boldsymbol{\nu}_{h}^{\mathbf{u}}\right|_{\mathrm{L}^{2}(\Omega)} \leqslant K_{1}\left|\boldsymbol{\nu}^{\mathbf{u}}\right|_{\mathrm{L}^{2}(\Omega)},
$$

and

$$
\int_{0}^{1} \tau\left(t_{f}-t \Delta t\right) d t \longrightarrow \tau\left(t_{f}\right)=0
$$

In conclusion, we proved that:

$$
\begin{gathered}
-\varrho_{0} \int_{0}^{t_{f}} \int_{\Omega} \mathbf{u} \cdot \boldsymbol{\nu}^{\mathbf{u}} d x \tau^{\prime} d t-\varrho_{0} \int_{\Omega} \mathbf{u}^{0} \cdot \boldsymbol{\nu}^{\mathbf{u}} d x \tau(0) \\
+\int_{0}^{t_{f}} \int_{\Omega} \frac{1}{2} \varrho_{0}\left[(\mathbf{u} \cdot \nabla) \mathbf{u} \cdot \boldsymbol{\nu}^{\mathbf{u}}-(\mathbf{u} \cdot \nabla) \boldsymbol{\nu}^{\mathbf{u}} \cdot \mathbf{u}\right]+2 \eta(\mathbf{c}) D \mathbf{u}: D \boldsymbol{\nu}^{\mathbf{u}} d x \tau(t) d t \\
\quad=\int_{0}^{t_{f}} \int_{\Omega} \varrho_{0} \mathbf{g} \cdot \boldsymbol{\nu}^{\mathbf{u}} d x \tau(t) d t-\int_{0}^{t_{f}} \int_{\Omega} \sum_{j=1}^{3}\left(\underline{c}_{j}-\alpha_{j}\right) \nabla \mu_{j} \cdot \boldsymbol{\nu}^{\mathbf{u}} d x \tau(t) d t .
\end{gathered}
$$

To finish, passing to the limit in the constraint equation yields:

$$
\operatorname{div}(\mathbf{u})=0 .
$$


6. Conclusion. We proposed in this article an original scheme for the discretization of the triphasic Cahn-Hilliard/Navier-Stokes model.

This scheme is unconditionally stable and preserves, at the discrete level, the main properties of the model, that is the volume conservation and the fact that the sum of the three order parameters remains equal to 1 during the time evolution.

We proved the existence of at least one solution of the discrete problem and, in the homogeneous case (i.e. three phases with the same densities), we proved the convergence of discrete solutions towards a weak solution of the model (whose existence is proved in the same time).

The main perspective is the study of the convergence in the case where the three fluids in presence have different densities. Even if the energy estimate (and the existence of discrete solutions) are still true in this case, it is delicate to obtain sufficient estimates which would lead, by compactness, to strong convergence on the velocity which is necessary to pass to the limit in non linear terms. Indeed, the Navier-Stokes equation involves a term of the form $\mathbf{u} \partial_{t} \varrho$. The time derivative of the density is not very smooth since it is a function of order parameters whose time derivative is in $\mathrm{L}^{2}\left(0, t_{f},\left(\mathrm{H}^{1}(\Omega)\right)^{\prime}\right)$.

\section{REFERENCES}

[1] D. M. Anderson, G. B. McFadden, And A. A. Wheeler, Diffuse-interface methods in fluid mechanics, in Annual review of fluid mechanics, Vol. 30, vol. 30 of Annu. Rev. Fluid Mech., Annual Reviews, Palo Alto, CA, 1998, pp. 139-165.

[2] F. BOYER, A theoretical and numerical model for the study of incompressible mixture flows, Comput. Fluids, 31 (2002), pp. 41-68.

[3] F. Boyer And P. FABRIE, Éléments d'analyse pour l'étude de quelques modèles d'écoulements de fluides visqueux incompressibles, vol. 52 of Mathématiques \& Applications (Berlin) [Mathematics \& Applications], Springer-Verlag, Berlin, 2006.

[4] F. Boyer And C. LapUerta, Study of a three component Cahn-Hilliard flow model, M2AN, 40 (2006), pp. 653-687.

[5] F. Boyer, C. Lapuerta, S. Minjeaud, and B. Piar, A local adaptive refinement method with multigrid preconditionning illustrated by multiphase flows simulations, ESAIM Proceedings, 27 (2009), pp. 15-53.

[6] F. Boyer, C. Lapuerta, S. Minjeaud, B. Piar, and M. Quintard, Cahn-Hilliard/NavierStokes model for the simulation of three-phase flows, Transp. Porous Media, 82 (2010), pp. $463-483$.

[7] F. Boyer and S. Minjeaud, Numerical schemes for a three component Cahn-Hilliard model, M2AN, 45 (2011), pp. 697-738.

[8] S. C. Brenner and L. R. Scott, The mathematical theory of finite element methods, vol. 15 of Texts in Applied Mathematics, Springer, New York, third ed., 2008.

[9] A. J. Chorin, Numerical solution of the Navier-Stokes equations, Mathematics of Computation, 22 (1968), pp. $745-762$.

[10] K. Deimling, Nonlinear functional analyis, Springer-Verlag, 1985.

[11] Charles M. Elliott and Harald Garcke, Diffusional phase transitions in multicomponent systems with a concentration dependent mobility matrix, Phys. D, 109 (1997), pp. 242-256.

[12] X. Feng, Fully discrete finite element approximations of the Navier-Stokes-Cahn-Hilliard diffuse interface model for two-phase fluid flows, SIAM J. Numer. Anal., 44 (2006), pp. 10491072.

[13] D. Fuster, G. Agbaglah, C. Josserand, S. Popinet, and S. Zaleski, Numerical simulation of droplets, bubbles and waves: state of the art, Fluid Dynamics Research, 41 (2009).

[14] D. E. Fyfe, E. S. ORAn, AND M. J. FRITTS, Surface tension and viscosity with lagrangian hydrodynamics on a triangular mesh, Journal of Computational Physics, 76 (1988), pp. 349384.

[15] J.-L. Guermond and L. Quartapelle, A projection FEM for variable density incompressible flows, J. Comput. Phys., 165 (2000), pp. 167-188.

[16] M. Herrmann, A balanced force refined level set grid method for two-phase flows on unstructured flow solver grids, Journal of Computational Physics, 227 (2008), pp. 2674-2706. 
[17] D. JACQmin, Calculation of two-phase Navier-Stokes flows using phase-field modeling, J. Comput. Phys., 155 (1999), pp. 96-127.

[18] D. Kay, V. Styles, And R. Welford, Finite element approximation of a Cahn-HilliardNavier-Stokes system, Interfaces Free Bound., 10 (2008), pp. 15-43.

[19] J. Kim, K. Kang, and J. Lowengrub, Conservative multigrid methods for ternary CahnHilliard systems, Commun. Math. Sci., 2 (2004), pp. 53-77.

[20] C. Liu And J. Shen, A phase field model for the mixture of two incompressible fluids and its approximation by a Fourier-spectral method, Phys. D, 179 (2003), pp. 211-228.

[21] C. LiU And N. J. Walkington, Convergence of numerical approximations of the incompressible Navier-Stokes equations with variable density and viscosity, SIAM J. Numer. Anal., 45 (2007), pp. 1287-1304 (electronic).

[22] PELICANS. Collaborative Development environment: https://gforge.irsn.fr/gf/project/ pelicans/.

[23] J. M. Seiler And K. Froment, Material effects on multiphase phenomena in late phases of severe accidents of nuclear reactors, Multiphase Science and Technologie, 12 (2000), pp. $117-257$.

[24] J. Simon, Compact sets in the space $L^{p}(0, T ; B)$, Ann. Mat. Pura Appl. (4), 146 (1987), pp. 6596.

[25] D. J. Torres and J. U. BrackBill, The point-set method: Front-tracking without connectivity, Journal of Computational Physics, 165 (2000), pp. 620-644.

[26] J. VAN KAN, A second-order accurate pressure correction scheme for viscous incompressible flow, SIAM Journal on Scientific and Statistical Computing, 7 (1986), pp. 870-891. 